\title{
Stochastic Synchronization of Reaction-Diffusion Neural Networks under General Impulsive Controller with Mixed Delays
}

\author{
Xinsong Yang, ${ }^{1}$ Chuangxia Huang, ${ }^{2}$ and Zhichun Yang ${ }^{1}$ \\ ${ }^{1}$ Department of Mathematics, Chongqing Normal University, Chongqing 400047, China \\ 2 Department of Mathematics, College of Mathematics and Computing Science, \\ Changsha University of Science and Technology, Changsha, Hunan 410076, China
}

Correspondence should be addressed to Xinsong Yang, xinsongyang@163.com

Received 23 September 2012; Accepted 25 November 2012

Academic Editor: Sabri Arik

Copyright (C) 2012 Xinsong Yang et al. This is an open access article distributed under the Creative Commons Attribution License, which permits unrestricted use, distribution, and reproduction in any medium, provided the original work is properly cited.

\begin{abstract}
This paper investigates drive-response synchronization of a class of reaction-diffusion neural networks with time-varying discrete and distributed delays via general impulsive control method. Stochastic perturbations in the response system are also considered. The impulsive controller is assumed to be nonlinear and has multiple time-varying discrete and distributed delays. Compared with existing nondelayed impulsive controller, this general impulsive controller is more practical and essentially important since time delays are unavoidable in practical operation. Based on a novel impulsive differential inequality, the properties of random variables and Lyapunov functional method, sufficient conditions guaranteeing the global exponential synchronization in mean square are derived through strict mathematical proof. In our synchronization criteria, the distributed delays in both continuous equation and impulsive controller play important role. Finally, numerical simulations are given to show the effectiveness of the theoretical results.
\end{abstract}

\section{Introduction}

Since the pioneering work of Pecora and Carroll [1], the issue of synchronization and chaos control has been extensively studied [2] due to its potential engineering applications such as secure communication, biological systems, and information processing (see [3-10]). It is shown that neural networks exhibit chaotic behavior and provided that parameters and delays are appropriately chosen (see $[11,12])$. Therefore, in recent years, synchronization and control of neural networks has been one of the hot research topics (see [13-15], etc.).

It is known that many pattern formation and wave propagation phenomena that appear in nature can be described by systems of coupled nonlinear differential equations, 
generally known as reaction-diffusion equations. These wave propagation phenomena are exhibited by systems belonging to very different scientific disciplines. The reaction-diffusion effects, therefore, cannot be neglected in both biological and man-made neural networks, especially when electrons are moving in noneven electromagnetic field [16]. So we must consider that the activations vary in space as well as in time, and in this case the model should be expressed by partial differential equations. There are some published papers concerning stability or synchronization of neural networks with reaction-diffusion terms and delays (see [17-25]). In [22], the authors investigated synchronization of reaction-diffusion neural networks with discrete and unbounded distributed delays. In [24], the authors investigated the boundedness and exponential stability for nonautonomous fuzzy cellular neural networks with unbounded distributed delays and reaction-diffusion terms. The authors of [25] studied exponential stability of reaction-diffusion Cohen-Grossberg neural networks with time-varying discrete delays and stochastic perturbations.

Time delays usually exist in neural networks due to finite speeds of switching of amplifiers and transmission of signals in hardware implementation. Ignoring them when studying dynamics of neural networks may lead to impractical results. Moreover, delays are commonly time varying and unknown [26]. Therefore, papers concerning synchronization or stability of neural networks with or without reaction-diffusion terms have considered various time delays. The authors in [11] studied exponential synchronization problem for coupled neural networks with constant time delay. In [27], both constant and time-varying discrete delays were considered for the synchronization of a class of delayed neural networks. In [28-31] several types of synchronization for neural networks with discrete and bounded distributed delays were studied. However, the delay kernel of the bounded distributed delays in [28-31] has to be 1 because the well-known Jensen's inequality [32] is not applicable anymore if the delay kernel is not 1 . In the case of unbounded distributed delay, it is necessary to consider the delay kernel, which satisfies the condition that its integral from zero to infinite is bounded $[22,33,34]$. But the authors in $[22,33,34]$ had to use algebraic approach instead of matrix method to derive their main results which has more complex form and is more conservative than those obtained by matrix method. In [21], Wang and Zhang studied global asymptotic stability of reaction-diffusion Cohen-Grossberg neural networks with unbounded distributed delays by using a matrix decomposition method, and the obtained results were in terms of linear matrix inequality (LMI). But the Lyapunov functional and proof process used in [21] are relatively complex. Recently, authors in [35] studied global asymptotic synchronization in an array of coupled neural networks with probabilistic interval timevarying coupling delays and unbounded distributed delays; a novel integral inequality including the Jensen's inequality as a special case was developed. By using the developed integral inequality, one can use LMI method to solve the problem of distributed delays with not-equal-to-1 delay kernel instead of the matrix decomposition method used in [21].

It should be noted that control method is of great significance to realize synchronization. Specially, in [29], the output feedback controller which has time-varying discrete and distributed delays was considered. On the other hand, impulsive control, as one of the most effective and economic control methods, has recently attracted great interests of many researchers in different fields, since it needs small control gains and acts only at discrete times, thus control cost and the amount of transmitted information can be reduced drastically (see $[3,9,26,36-40]$ and references cited therein). As for neural networks with reaction-diffusion terms, there are several results on synchronization via control. For instance, state feedback control technique is utilized in [20] to realize exponential synchronization of stochastic fuzzy cellular neural networks with time delay in the leakage term and reaction diffusion. In [22], 
global exponential stability and synchronization of delayed reaction-diffusion neural networks under hybrid state feedback control and impulsive control. However, to the authors knowledge, impulsive control has not been considered in the literature to realize synchronization of reaction-diffusion neural networks. Moreover, the impulsive controllers in $[3,9,36-40]$ were nondelayed. Recently, in [41], global exponential stability of fuzzy reactiondiffusion cellular neural networks with time-varying discrete delays and unbounded distributed delays and impulsive perturbations were studied. Nevertheless, to the best of our knowledge, there are no results on stability or synchronization of reaction-diffusion neural networks with time-varying discrete delays and distributed delays under impulsive controller which has multiple time-varying delays, let alone impulsive controller with distributed delays. If these delays are considered in impulsive controller, the analysis methods used in $[3,9,26,36-40]$ are not applicable anymore. Considering the fact that both discrete delays and distributed delays are unavoidable in practice, it is of great importance to consider delayed impulsive control to synchronize-delayed neural neural networks.

Being motivated by the above discussions, this paper aims to study the global exponential derive-response synchronization of reaction-diffusion neural networks with multiple time-varying discrete delays and unbounded distributed delays via general impulsive control. The general impulsive controller is assumed to be nonlinear and has multiple timevarying discrete and distributed delays. Since time delays are always vary and unavoidable in practical operation, the general impulsive controller is essentially important and more practical than existing nondelayed impulsive controller. Stochastic perturbations in the response system are also considered. By using a novel integral inequality in [35], the problem of distributed delays with not-equal-to-1 delay kernel can be solved by matrix method. By utilizing the novel integral inequality, the properties of random variables and Lyapunov functional method, sufficient conditions guaranteeing the considered drive-response systems to realize synchronization in mean square are derived through strict mathematical proof. The proof process and the results are very simple. Finally, numerical simulations are given to show the effectiveness of the theoretical results.

The rest of this paper is organized as follows. In Section 2, the considered model of coupled reaction-diffusion neural networks with delays is presented. Some necessary assumptions, definitions, and lemmas are also given in this section. In Section 3, synchronization for the proposed model is studied. Then, in Section 4, simulation examples are presented to show the effectiveness of the theoretical results. Finally, Section 5 provides some conclusions.

Notations. In the sequel, if not explicitly stated, matrices are assumed to have compatible dimensions. $\mathbb{N}_{+}$denotes the set of positive integers. $I_{n}$ denotes the $n \times n$ identity matrix. $\mathbb{R}^{n}$ denotes the Euclidean space, and $\mathbb{R}^{n \times m}$ is the set of all $n \times m$ real matrix. $\lambda_{\max }(A)$ and $\lambda_{\min }(A)$ mean the largest and smallest eigenvalues of matrix $A$, respectively, $\|A\|=$ $\sqrt{\lambda_{\max }\left(A^{T} A\right)}$, where $T$ denotes transposition. $C=\operatorname{diag}\left(c_{1}, c_{2}, \ldots, c_{n}\right)$ means $C$ is a diagonal matrix. Moreover, let $\left(S, \mathcal{F},\left\{\mathcal{F}_{t}\right\}_{t>0}, P\right)$ be a complete probability space with filtration $\left\{\mathcal{F}_{t}\right\}_{t>0}$ satisfying the usual conditions (i.e., the filtration contains all $P$-null sets and is right continuous). Denote by $L_{q_{0}}^{P}\left((-\infty, 0] ; \mathbb{R}^{n}\right)$ the family of all $\mathcal{F}_{0}$-measurable $C\left((-\infty, 0] ; \mathbb{R}^{n}\right)$ valued random variables $\xi=\{\xi(s): s \leq 0\}$ such that $\sup _{s \leq 0} \mathbb{E}\left(\|\xi(s)\|^{p}\right)<\infty$, where $\mathbb{E}\{\cdot\}$ stands for mathematical expectation operator with respect to the given probability measure $P$. Sometimes, the arguments of a function or a matrix will be omitted in the analysis when no confusion can arise. 


\section{Preliminaries}

Consider a delayed neural network with reaction-diffusion terms which is described as follows:

$$
\begin{aligned}
\frac{\partial y_{i}(t, x)}{\partial t}= & \sum_{l=1}^{m} \frac{\partial}{\partial x_{l}}\left(r_{i l} \frac{\partial y_{i}(t, x)}{\partial x_{l}}\right)-c_{i} y_{i}(t, x)+\sum_{j=1}^{n} a_{i j} f_{j}\left(y_{j}(t, x)\right) \\
& +\sum_{j=1}^{n} b_{i j} f_{j}\left(y_{j}\left(t-\tau_{1}(t), x\right)\right)+\sum_{j=1}^{n} d_{i j} \int_{-\infty}^{t} K(t-s) f_{j}\left(y_{j}(s, x)\right) \mathrm{d} s+I_{i}(t)
\end{aligned}
$$

or in a compact form

$$
\begin{aligned}
\frac{\partial y(t, x)}{\partial t}= & \sum_{l=1}^{m} \frac{\partial}{\partial x_{l}}\left(R_{l} \frac{\partial y(t, x)}{\partial x_{l}}\right)-C y(t, x)+A f(y(t, x))+B f\left(y\left(t-\tau_{1}(t), x\right)\right) \\
& +D \int_{-\infty}^{t} K(t-s) f(y(s, x)) \mathrm{d} s+I(t)
\end{aligned}
$$

where $i=1,2, \ldots, n, R_{l}=\operatorname{diag}\left(r_{1 l}, r_{2 l}, \ldots, r_{n l}\right), l=1,2, \ldots, m, r_{i l} \geq 0$ means the transmission diffusion coefficient along the $i$ th neuron; $x=\left(x_{1}, x_{2}, \ldots, x_{m}\right)^{T} \in \Omega \subset \mathbb{R}^{m}, \Omega=\{x \mid$ $\left.\left|x_{k}\right| \leq z_{l}, l=1,2, \ldots, m\right\}$, and $z_{l}$ is a constant. $y(t, x)=\left(y_{1}(t, x), y_{2}(t, x), \ldots, y_{n}(t, x)\right)^{T} \in \mathbb{R}^{n}$ represents the state vector of the network at time $t$ and in space $x ; n$ corresponds to the number of neurons; $f(y(t, x))=\left(f_{1}\left(y_{1}(t, x)\right), \ldots, f_{n}\left(y_{n}(t, x)\right)\right)^{T}$ is the neuron activation function at time $t$ and in space $x ; C=\operatorname{diag}\left(c_{1}, c_{2}, \ldots, c_{n}\right)$ with $c_{i}>0 ; A=\left(a_{i j}\right)_{n \times n}, B=\left(b_{i j}\right)_{n \times n}$ and $D=\left(d_{i j}\right)_{n \times n}$ are the connection weight matrix; $I(t)=\left(I_{1}(t), I_{2}(t), \ldots, I_{n}(t)\right)^{T} \in \mathbb{R}^{n}$ is an external input vector. The bounded function $\tau_{1}(t)$ represents unknown time-varying discrete delay of the system with $0<\tau_{1}(t) \leq \bar{\tau}_{1}$, in which $\bar{\tau}_{1}$ is a constant, $K(t)$ is a nonnegative bounded scalar function defined on $[0,+\infty)$ describing the delay kernel of the unbounded distributed delay.

We suppose that system (2.2) has an unique continuous solution for any initial condition of the following form: $y(s, x)=\phi(s, x) \in C\left([-\infty, 0] \times \Omega, \mathbb{R}^{n}\right)$, where $C\left([-\infty, 0] \times \Omega, \mathbb{R}^{n}\right)$ denotes the Banach space of all continuous functions from $[-\infty, 0] \times \Omega$ to $\mathbb{R}^{n}$ with the norm

$$
\|\phi(s, x)\|=\left[\int_{\Omega} \phi^{T}(s, x) \phi(s, x) \mathrm{d} x\right]^{1 / 2} .
$$

It is assumed that (2.2) satisfies the following Dirichlet boundary condition:

$$
y(t, x)=0, \quad(t, x) \in[-\infty,+\infty] \times \partial \Omega
$$


Based on the concept of drive-response synchronization, we take (2.2) as the driver system and design the following controlled response system:

$$
\begin{aligned}
\mathrm{d} u(t, x)= & {\left[\sum_{l=1}^{m} \frac{\partial}{\partial x_{l}}\left(R_{l} \frac{\partial u(t, x)}{\partial x_{l}}\right)-C u(t, x)+A f(u(t, x))+B f\left(u\left(t-\tau_{1}(t), x\right)\right)\right.} \\
& \left.+D \int_{-\infty}^{t} K(t-s) f(u(s, x)) \mathrm{d} s+I(t)+\sum_{k=1}^{+\infty} \delta\left(t-t_{k}\right) U_{k}(t, x)\right] \mathrm{d} t+\sigma(t, x) \mathrm{d} \omega(t),
\end{aligned}
$$

where $e(t, x)=u(t, x)-y(t, x), \delta(t)$ is the Dirac delta function, the time sequence $\left\{t_{k}\right\}$ satisfies $0=t_{0}<t_{1}<t_{2}<\cdots<t_{k-1}<t_{k}<\cdots$, and $\lim _{k \rightarrow+\infty} t_{k}=+\infty$. $U_{k}(t, x)$ is the control input. $\omega(t)=\left(\omega_{1}(t), \ldots, \omega_{n}(t)\right)^{T} \in \mathbb{R}^{n}$ is a $n$-dimensional Brown motion defined on $\left(S, \mathcal{F},\left\{\mathcal{F}_{t}\right\}_{t \geq 0}, P\right)$. Here, the white noise $\mathrm{d} \omega_{i}(t)$ is independent of $\mathrm{d} \omega_{j}(t)$ for $i \neq j$, and $\sigma(t, x) \triangleq$ $\sigma\left(t, e(t, x), e\left(t-\tau_{2}(t), x\right), \int_{t-\tau_{3}(t)}^{t} e(s, x) \mathrm{d} s\right)$ is the noise intensity function matrix, in which the bounded functions $\tau_{2}(t)$ and $\tau_{3}(t)$ represent unknown discrete and distributed delays of the system in the stochastic perturbation with $0<\tau_{i}(t) \leq \bar{\tau}_{i}, i=2,3$. This type of stochastic perturbation can be regarded as a result from the occurrence of random uncertainties during the process of transmission. We assume that the output signals of (2.2) can be received by (2.5).

In the present paper, the control input $U_{k}(t, x)$ is assumed to be the following form:

$$
U_{k}(t, x)=h_{k}\left(e(t, x), e\left(t-\eta_{1}(t), x\right), \ldots, e\left(t-\eta_{q}(t), x\right), \int_{t-\eta_{q+1}(t)}^{t} e(s, x) \mathrm{d} s\right)-e(t, x),
$$

where $\eta_{i}(t) i=1,2, \ldots, q+1$ are unknown time-varying delays with $0<\eta_{i}(t) \leq \bar{\eta}_{i}$.

Integrating from $t_{k}-\varepsilon$ to $t_{k}+\varepsilon$ ( $\varepsilon>0$ is a sufficient small constant) on both sides of system (2.5) and letting $\varepsilon \rightarrow 0^{+}$, one gets from the property of the Dirac delta function that

$$
\begin{aligned}
u\left(t_{k}^{+}, x\right)-u\left(t_{k}^{-}, x\right)= & h_{k}\left(e\left(t_{k}, x\right), e\left(t_{k}-\eta_{1}\left(t_{k}\right), x\right), \ldots, e\left(t_{k}-\eta_{q}\left(t_{k}\right), x\right), \int_{t_{k}-\eta_{q+1}\left(t_{k}\right)}^{t_{k}} e(s, x) \mathrm{d} s\right) \\
& -e\left(t_{k}, x\right),
\end{aligned}
$$

where $u\left(t_{k^{\prime}}^{+}, x\right)=\lim _{t \rightarrow t_{k}^{+}} u(t, x), u\left(t_{k^{\prime}}^{-}, x\right)=\lim _{t \rightarrow t_{k}^{-}} u(t, x)$. In the following, we use $h_{k}\left(t_{k}, x\right)$ to denote $h_{k}\left(e\left(t_{k}, x\right), e\left(t_{k}-\eta_{1}\left(t_{k}\right), x\right), \ldots, e\left(t_{k}-\eta_{q}\left(t_{k}\right), x\right), \int_{t_{k}-\eta_{q+1}\left(t_{k}\right)}^{t_{k}} e(s, x) \mathrm{d} s\right)$ for short.

Remark 2.1. Equation (2.7) is actually the impulsive controller of response system (2.5). To the best of our knowledge, result on synchronization of reaction-diffusion neural networks under impulsive control is seldom. In [22], global exponential synchronization of delayed reactiondiffusion neural networks was studied. However, the control scheme in [22] is hybrid nondelayed state feedback control and nondelayed impulsive control, and the continuous state feedback controller is indispensable. Moreover, the impulsive controller (2.7) is very general, since it includes information of multiple time-varying discrete delays and time-varying distributed delays. Nevertheless, most of published paper concerning impulsive control 
including $[3,9,26,36-40]$ did not consider time delay in the impulsive function, let alone multiple time-varying discrete delays and time-varying distributed delays. It is known that both discrete delays and distributed delays are unavoidable and often time-varying in neural networks, hence considering impulsive control with time-varying discrete delays and timevarying distributed delays is essentially important. However, when time-varying discrete delays and time-varying distributed delays are considered in impulsive control, the results in $[3,9,26,36-40]$ is not applicable anymore.

From (2.7), the controlled system (2.5) can be rewritten as

$$
\begin{gathered}
\mathrm{d} u(t, x)=\left[\sum_{l=1}^{m} \frac{\partial}{\partial x_{l}}\left(R_{l} \frac{\partial u(t, x)}{\partial x_{l}}\right)-C u(t, x)+A f(u(t, x))+B f\left(u\left(t-\tau_{1}(t), x\right)\right)\right. \\
\left.\quad+D \int_{-\infty}^{t} K(t-s) f(u(s, x)) \mathrm{d} s+I(t)\right] \mathrm{d} t+\sigma(t, x) \mathrm{d} \omega(t), \quad t \neq t_{k}, \\
u\left(t_{k}^{+}, x\right)=u\left(t_{k}^{-}, x\right)+h_{k}\left(t_{k}, x\right)-e\left(t_{k}, x\right), \quad t=t_{k}, \quad k \in \mathbb{N}_{+} .
\end{gathered}
$$

To maintain consistency with above definitions, the initial value and the boundary condition of (2.8) are given in the following form:

$$
\begin{aligned}
& u(s, x)=\varphi(s, x) \in C\left([-\infty, 0] \times \Omega, \mathbb{R}^{n}\right), \\
& u(t, x)=0, \quad(t, x) \in[-\infty,+\infty] \times \partial \Omega .
\end{aligned}
$$

Throughout this paper, we always assume that $u(t, x)$ is left continuous at $t_{k}$, that is, $u\left(t_{k}^{-}, x\right)=u\left(t_{k}, x\right)$. Then subtracting (2.2) from (2.8) gets the following error dynamical system:

$$
\begin{gathered}
\mathrm{d} e(t, x)=\left[\sum_{l=1}^{m} \frac{\partial}{\partial x_{l}}\left(R_{l} \frac{\partial e(t, x)}{\partial x_{l}}\right)-C e(t, x)+A g(e(t, x))+B g\left(e\left(t-\tau_{1}(t), x\right)\right)\right. \\
\left.+D \int_{-\infty}^{t} K(t-s) g(e(s, x)) \mathrm{d} s\right] \mathrm{d} t+\sigma(t, x) \mathrm{d} \omega(t), \quad t \neq t_{k}, \\
e\left(t_{k}^{+}, x\right)=h_{k}\left(t_{k}, x\right), \quad t=t_{k}, k \in \mathbb{N}_{+}
\end{gathered}
$$

where $g(e(t, x))=f(u(t, x))-f(y(t, x))$.

It is obvious that system (2.11) satisfies the Dirichlet boundary condition, and its initial condition is

$$
e(s, x)=\varphi(s, x)-\phi(s, x)=\bar{\varphi}(s, x) \in C\left([-\infty, 0] \times \Omega, \mathbb{R}^{n}\right), \quad i=1,2, \ldots, N
$$

It is easy to see that the error system (2.11) admits a zero solution. Clearly, if the zero solution is globally exponentially stable, then the controlled system (2.8) is globally exponentially synchronized with system (2.2). 
Throughout this paper, we assume that

$\left(\mathrm{H}_{1}\right)$ for any $u, v \in \mathbb{R}$, there exist constants $\mu_{i}(i=1,2, \ldots, n)$ such that $\left|f_{i}(u)-f_{i}(v)\right| \leq$ $\mu_{i}|u-v| ;$

$\left(\mathrm{H}_{2}\right)$ there is a positive constant $\bar{k}$ such that $\int_{0}^{+\infty} K(u) \mathrm{d} u=\bar{k}$;

$\left(\mathrm{H}_{3}\right)$ there exist positive constants $\rho_{1}, \rho_{2}$ and $\rho_{3}$ such that

$$
\begin{aligned}
\operatorname{trace}\left[\sigma^{T}(t, x) \sigma(t, x)\right] \leq & \rho_{1} e^{T}(t, x) e(t, x)+\rho_{2} e^{T}\left(t-\tau_{2}(t), x\right) e\left(t-\tau_{2}(t), x\right) \\
& +\rho_{3} \int_{t-\tau_{3}(t)}^{t} e^{T}(s, x) e(s, x) \mathrm{d} s
\end{aligned}
$$

$\left(\mathrm{H}_{4}\right)$ there exist nonnegative constants $\alpha_{k}, \beta_{k^{\prime}}^{j}, j=1,2, \ldots, q+1$ such that

$$
\begin{aligned}
h_{k}^{T}\left(t_{k}, x\right) h_{k}\left(t_{k}, x\right) \leq & \alpha_{k} e^{T}\left(t_{k}, x\right) e\left(t_{k}, x\right)+\beta_{k}^{1} e^{T}\left(t_{k}-\eta_{1}\left(t_{k}\right), x\right) e\left(t_{k}-\eta_{1}\left(t_{k}\right), x\right) \\
& +\cdots+\beta_{k}^{q} e^{T}\left(t_{k}-\eta_{q}\left(t_{k}\right)\right) e\left(t_{k}-\eta_{q}\left(t_{k}\right)\right)+\beta_{k}^{q+1} \int_{t_{k}-\eta_{q+1}\left(t_{k}\right)}^{t_{k}} e^{T}(s, x) e(s, x) \mathrm{d} s .
\end{aligned}
$$

The following basic definitions and lemmas are needed in this paper to get main results.

Definition 2.2 (see [9]). The dynamical network (2.9) is said to be globally exponentially synchronized with system (2.2) in mean square if there exist constants $M>1$ and $\theta>0$ such that for any initial values (2.12)

$$
\mathbb{E}\left\{\|e(t, x)\|^{2}\right\} \leq \max _{s \leq 0} \mathbb{E}\left\{\|\bar{\varphi}(s, x)\|^{2}\right\} M e^{-\theta t}
$$

hold for $t \geq 0$.

Lemma 2.3 (see [17]). Let $\Omega$ be a cube $\left|x_{k}\right|<l_{k}(k=1,2, \ldots, m)$, and let $v(x)$ be a real-valued function belonging to $C^{1}(\Omega)$ which vanish on the boundary $\partial \Omega$ of $\Omega$, that is, $\left.v(x)\right|_{\partial \Omega}=0$. Then

$$
\int_{\Omega} v^{2}(x) \mathrm{d} x \leq l_{k}^{2} \int_{\Omega}\left|\frac{\partial v(x)}{\partial x_{k}}\right|^{2} \mathrm{~d} x .
$$

Lemma 2.4 (see [42]). If $X, Y$ are real matrices with appropriate dimensions, then there exist number $\varepsilon>0$ such that

$$
X^{T} Y+Y^{T} X \leq \varepsilon X^{T} Y+\frac{1}{\varepsilon} Y^{T} Y
$$


Lemma 2.5 (see [35]). Suppose that $K(t)$ is a nonnegative bounded scalar function defined on $[0,+\infty)$, and there exists a positive constant $k$ such that $\int_{0}^{+\infty} K(u) \mathrm{d} u=k$. For any constant matrix $D \in \mathbb{R}^{n \times n}, D>0$, and vector function $x:(-\infty, t] \rightarrow \mathbb{R}^{n}$ for $t \geq 0$, one has

$$
k \int_{-\infty}^{t} K(t-s) x^{T}(s) D x(s) \mathrm{d} s \geq\left(\int_{-\infty}^{t} K(t-s) x(s) \mathrm{d} s\right)^{T} D \int_{-\infty}^{t} K(t-s) x(s) \mathrm{d} s
$$

provided the integrals are all well defined.

Remark 2.6. When there is a positive bounded function $k(t)$ such that $\int_{0}^{\theta(t)} K(u) \mathrm{d} u=k(t)$, where $0<\theta(t) \leq \theta$, then the inequality (2.18) becomes the following from:

$$
k(t) \int_{t-\theta(t)}^{t} K(t-s) x^{T}(s) D x(s) \mathrm{d} s \geq\left(\int_{t-\theta(t)}^{t} K(t-s) x(s) \mathrm{d} s\right)^{T} D \int_{t-\theta(t)}^{t} K(t-s) x(s) \mathrm{d} s .
$$

Specially, when $K(t)=1$ for $t \geq 0$, then $k(t)=\theta(t)$ in (2.19). In this case, the inequality (2.19) turns out to the well-known Jensen's inequality [32]. In the literature, there were many results concerning stability or synchronization of neural networks with bounded distributed delays, for instance, see [28-31]. However, the delay kernels in [28-31] were all assumed to be 1. Obviously, the unbounded distributed delays in this paper include those [28-31] as a special case. It is easy to see from inequalities (2.18) and (2.19) that results of this paper are also applicable to neural networks with bounded distributed delays, no matter whether $K(t)$ is equal to 1 or not. In this sense, models in this paper are more general than those those in [28-31].

Lemma 2.7. Consider the following impulsive differential inequalities:

$$
\begin{gathered}
D^{+} v(t) \leq a v(t)+b_{1}[v(t)]_{\tau_{1}}+b_{2}[v(t)]_{\tau_{2}}+\cdots+b_{m}[v(t)]_{\tau_{m}}, \quad t \neq t_{k}, \quad t \geq t_{0}, \\
v\left(t_{k}^{+}\right) \leq p_{k} v\left(t_{k}^{-}\right)+q_{k}^{1}\left[v\left(t_{k}^{-}\right)\right]_{\tau_{1}}+q_{k}^{2}\left[v\left(t_{k}^{-}\right)\right]_{\tau_{2}}+\cdots+q_{k}^{m}\left[v\left(t_{k}^{-}\right)\right]_{\tau_{m}}, \quad k \in \mathbb{N}_{+}, \\
v(t)=\phi(t), \quad t \in\left[t_{0}-\tau, t_{0}\right],
\end{gathered}
$$

where $a, b_{i}, p_{k}, q_{k^{\prime}}^{i}$ and $\tau_{i}$ are constants, $b_{i} \geq 0, p_{k} \geq 0, q_{k}^{i} \geq 0, \tau_{i} \geq 0, i=1,2, \ldots, m, v(t) \geq 0$, $[v(t)]_{\tau_{i}}=\sup _{t-\tau_{i} \leq s \leq t} v(s),\left[v\left(t_{k}^{-}\right)\right]_{\tau_{i}}=\sup _{t_{k}-\tau_{i}\left(t_{k}\right) \leq s<t_{k}} v(s), \phi(t)$ is continuous on $\left[t_{0}-\tau, t_{0}\right]$, and $v(t)$ is continuous except $t_{k}, k \in \mathbb{N}_{+}$, where it has jump discontinuities. The consequence $\left\{t_{k}\right\}$ satisfies $0=t_{0}<t_{1}<t_{2}<\cdots<t_{k}<t_{k+1}<\cdots$, and $\lim _{k \rightarrow+\infty} t_{k}=+\infty$. Suppose that

$$
\begin{gathered}
p_{k}+\sum_{i=1}^{m} q_{k}^{i}<1 \\
a+\frac{\sum_{i=1}^{m} b_{i}}{p_{k}+\sum_{j=1}^{m} q_{k}^{j}}+\frac{\ln \left(p_{k}+\sum_{j=1}^{m} q_{k}^{j}\right)}{t_{k+1}-t_{k}}<0 .
\end{gathered}
$$


Then there exist constants $\beta>1$ and $\lambda>0$ such that

$$
v(t) \leq\|\phi\|_{\tau} \beta e^{-\lambda\left(t-t_{0}\right)}, \quad t \geq t_{0}
$$

where $\|\phi\|_{\tau}=\sup _{t_{0}-\tau \leq s \leq t_{0}}\|\phi(s)\|, \tau=\max \left\{\tau_{i}, i=1,2, \ldots, m\right\}$.

The proof of Lemma 2.7 is given in the appendix, which is partly similarly to that of Lemma 1 in [43].

Remark 2.8. Lemma 2.7 actually provides stability criterion for impulsive differential equations with multiple time-varying delays, and impulsive function is related to the same multiple time-varying delays. Actually, Lemma 2.7 can be written in a more general form. Let $b_{i}=0, i=h+1, \ldots, m, q_{k}^{j}=0, j=1, \ldots, h, 1<h<m-1, \tau_{h+1}=\sigma_{1}, \ldots, \tau_{h+m-h}=\sigma_{m-h}=\sigma_{r}$, $q_{k}^{h+1}=\tilde{q}_{k}^{1}, \ldots, q_{k}^{h+m-h}=\tilde{q}_{k}^{m-h}=\tilde{q}_{k}^{r}$, the other parameters are the same as those in Lemma 2.7. Then one can get the following Lemma 2.9.

Lemma 2.9. Consider the following impulsive differential inequality:

$$
\begin{gathered}
D^{+} v(t) \leq a v(t)+b_{1}[v(t)]_{\tau_{1}}+b_{2}[v(t)]_{\tau_{2}}+\cdots+b_{h}[v(t)]_{\tau_{h^{\prime}}} \quad t \neq t_{k}, \quad t \geq t_{0}, \\
v\left(t_{k}^{+}\right) \leq p_{k} v\left(t_{k}^{-}\right)+\tilde{q}_{k}^{1}\left[v\left(t_{k}^{-}\right)\right]_{\sigma_{1}}+\tilde{q}_{k}^{2}\left[v\left(t_{k}^{-}\right)\right]_{\sigma_{2}}+\cdots+\tilde{q}_{k}^{r}\left[v\left(t_{k}^{-}\right)\right]_{\sigma_{r}}, \quad k \in \mathbb{N}_{+}, \\
v(t)=\phi(t), \quad t \in\left[t_{0}-\tau, t_{0}\right] .
\end{gathered}
$$

Suppose that

$$
p_{k}+\sum_{i=1}^{r} \tilde{q}_{k}^{i}<1, \quad a+\frac{\sum_{i=1}^{h} b_{i}}{p_{k}+\sum_{j=1}^{r} \tilde{q}_{k}^{j}}+\frac{\ln \left(p_{k}+\sum_{j=1}^{r} \tilde{q}_{k}^{j}\right)}{t_{k+1}-t_{k}}<0 .
$$

Then there exist constants $\beta>1$ and $\lambda>0$ such that

$$
v(t) \leq\|\phi\|_{\tau} \beta e^{-\lambda\left(t-t_{0}\right)}, \quad t \geq t_{0}
$$

where $\|\phi\|_{\tau}=\sup _{t_{0}-\tau \leq s \leq t_{0}}\|\phi(s)\|, \tau=\max \left\{\tau_{i}, \sigma_{j}, i=1,2, \ldots, h, j=1,2, \ldots, r\right\}$.

Remark 2.10. Lemmas 2.7 and 2.9 are general. Specially, if $\tilde{q}_{k}^{i}=0, i=1,2, \ldots, r$, then the inequalities in (2.25) becomes

$$
p_{k}<1, \quad a+\frac{\sum_{i=1}^{h} b_{i}}{p_{k}}+\frac{\ln p_{k}}{t_{k+1}-t_{k}}<0
$$

Take $p=\max \left\{p_{k}, k \in \mathbb{N}_{+}\right\}, \rho=\sup _{k \in \mathbb{N}^{+}}\left\{t_{k}-t_{k-1}\right\}$. Then $p<1$ and

$$
a+\frac{\sum_{i=1}^{h} b_{i}}{p_{k}}+\frac{\ln p_{k}}{t_{k+1}-t_{k}} \leq a+\frac{\sum_{i=1}^{h} b_{i}}{p_{k}}+\frac{\ln p}{t_{k+1}-t_{k}} \leq a+\frac{\sum_{i=1}^{h} b_{i}}{p_{k}}+\frac{\ln p}{\rho} .
$$


Therefore,

$$
p<1, \quad a+\frac{\sum_{i=1}^{h} b_{i}}{p}+\frac{\ln p}{\rho}<0
$$

implies (2.27); that is, the inequality (2.27) is less conservative than (2.29). In fact, the inequality (2.29) is exactly the inequalities (5) and (6) in Theorem 3.1 of [26]. (In the proof in Theorem 3.1 in [26], one can get from $b_{i}=\left(L_{i} / \alpha\right) \sqrt{\lambda_{\max }(P) / \lambda_{\min }(P)}$ that $\left(L_{i}^{2} \lambda_{\max }(P)\right) /\left(b_{i} \lambda_{\min }(P)\right)=\alpha L_{i} \sqrt{\lambda_{\max }(P) / \lambda_{\min }(P)}$. By comparing the coefficients in the first two inequalities in the proof of Theorem 3.1 in [26] with those in the inequalities (5) and (6) in [26], the conclusion can be easily achieved). Hence, Lemmas 2.7 and 2.9 improve and extend the Theorem 3.1 in [26]. In the literature, many results including those in $[3,9,38,40]$ were derived by using similar method used in [26]. Since Lemmas 2.7 and 2.9 include corresponding results in [26] as a special case and are less conservative than them, Lemmas 2.7 and 2.9 are very useful for stabilization and synchronization of impulsive control system.

\section{Main Results}

In this section, the global exponential synchronization criteria for system (2.8) and (2.2) are derived through strict mathematical reasoning.

Theorem 3.1. Suppose that conditions $\left(H_{1}\right)-\left(H_{4}\right)$ hold. If there exists constants $\varepsilon_{1}>0, \varepsilon_{2}>0$ and $\varepsilon_{3}>0$ such that

$$
\begin{gathered}
0<\alpha_{k}+\sum_{i=1}^{q} \beta_{k}^{i}+\beta_{k}^{q+1} \bar{\eta}_{q+1}<1, \quad k \in \mathbb{N}_{+} \\
a+\frac{\varepsilon_{2} \mu+\rho_{2}+\varepsilon_{3} \bar{k}^{2} \mu+\rho_{3} \bar{\tau}_{3}}{\alpha_{k}+\sum_{i=1}^{q} \beta_{k}^{i}+\beta_{k}^{q+1} \bar{\eta}_{q+1}}+\frac{\ln \left(\alpha_{k}+\sum_{i=1}^{q} \beta_{k}^{i}\right)+\beta_{k}^{q+1} \bar{\eta}_{q+1}}{t_{k+1}-t_{k}}<0,
\end{gathered}
$$

where $a=-2 \lambda_{\min }(\widetilde{R}+C)+\varepsilon_{1}^{-1}\|A\|^{2}+\varepsilon_{1} \mu+\varepsilon_{2}^{-1}\|B\|^{2}+\varepsilon_{3}^{-1}\|D\|^{2}+\rho_{1}, \widetilde{R}=\operatorname{diag}\left(\sum_{l=1}^{m}\left(r_{1 l} / z_{l}^{2}\right)\right.$, $\left.\sum_{l=1}^{m}\left(r_{2 l} / z_{l}^{2}\right), \ldots, \sum_{l=1}^{m}\left(r_{n l} / z_{l}^{2}\right)\right), \mu=\max \left\{\mu_{i}^{2}, i=1,2, \ldots, n\right\}$. Then, under the impulsive controller (2.7), the controlled system (2.8) is globally exponentially synchronized with system (2.2) in mean square.

Proof. Consider the following Lyapunov function:

$$
V(t)=\int_{\Omega} \frac{1}{2} e^{T}(t, x) e(t, x) \mathrm{d} x
$$

We use $\mathcal{L V}(t)$ to denote the infinitesimal operator of $V(t)$ [44], which is defined as

$$
\mathcal{L V}(t)=\lim _{\Delta \rightarrow 0^{+}} \Delta^{-1}[\mathbb{E}\{V(t+\Delta) \mid t\}-V(t)]
$$


Based on the property of Wiener process [11], differentiating $V(t)$ along the solution of the error system (2.11) for $t \in\left(t_{k-1}, t_{k}\right], k \in \mathbb{N}_{+}$obtains that

$$
\mathrm{d} V(t)=\mathcal{L}(t) \mathrm{d} t+e(t, x) \sigma(t, x) \mathrm{d} \omega(t),
$$

where

$$
\begin{aligned}
\mathcal{L} V(t)=\int_{\Omega}[ & e^{T}(t, x) \sum_{l=1}^{m} \frac{\partial}{\partial x_{l}}\left(R_{l} \frac{\partial e(t, x)}{\partial x_{l}}\right)-e^{T}(t, x) C e(t, x)+e^{T}(t, x) A g(e(t, x)) \\
& +e^{T}(t, x) B g\left(e\left(t-\tau_{1}(t), x\right)\right)+e^{T}(t, x) D \int_{-\infty}^{t} K(t-s) g(e(s, x)) \mathrm{d} s \\
& \left.+\frac{1}{2} \operatorname{trace}\left[\sigma^{T}(t, x) \sigma(t, x)\right]\right] \mathrm{d} x .
\end{aligned}
$$

From the Green's formula and the Dirichlet boundary condition, we have (see [17-19])

$$
\begin{aligned}
\int_{\Omega} e^{T}(t, x) \sum_{l=1}^{m} \frac{\partial}{\partial x_{l}}\left(R_{l} \frac{\partial e(t, x)}{\partial x_{l}}\right) \mathrm{d} x= & \int_{\Omega} \sum_{i=1}^{n} e_{i}(t, x) \sum_{l=1}^{m} \frac{\partial}{\partial x_{l}}\left(r_{i l} \frac{\partial e_{i}(t, x)}{\partial x_{l}}\right) \mathrm{d} x \\
= & \sum_{i=1}^{n} \int_{\Omega} e_{i}(t, x) \nabla\left(r_{i l} \frac{\partial e_{i}(t, x)}{\partial x_{l}}\right)_{l=1}^{m} \mathrm{~d} x \\
= & \sum_{i=1}^{n} \int_{\Omega} \nabla\left(e_{i}(t, x) r_{i l} \frac{\partial e_{i}(t, x)}{\partial x_{l}}\right)_{l=1}^{m} \mathrm{~d} x \\
& -\sum_{j=1}^{n} \int_{\Omega}\left(r_{i l} \frac{\partial e_{i}(t, x)}{\partial x_{l}}\right)_{l=1}^{m} \nabla e_{i}(t, x) \mathrm{d} x \\
= & \sum_{i=1}^{n} \int_{\partial \Omega}\left(e_{i}(t, x) r_{i l} \frac{\partial e_{i}(t, x)}{\partial x_{l}}\right)_{l=1}^{m} \mathrm{~d} x \\
& -\sum_{i=1}^{n} \int_{\Omega} \sum_{l=1}^{m} r_{i l}\left(\frac{\partial e_{i}(t, x)}{\partial x_{l}}\right)^{2} \mathrm{~d} x \\
= & -\sum_{i=1}^{n} \int_{\Omega} \sum_{l=1}^{m} r_{i l}\left(\frac{\partial e_{i}(t, x)}{\partial x_{l}}\right)^{2} \mathrm{~d} x,
\end{aligned}
$$

in which $\nabla=\left(\partial / \partial x_{1}, \partial / \partial x_{2}, \ldots, \partial / \partial x_{m}\right)$ is the gradient operator, and

$$
\left(r_{i l} \frac{\partial e_{i}(t, x)}{\partial x_{l}}\right)_{l=1}^{m}=\left(r_{i 1} \frac{\partial e_{i}(t, x)}{\partial x_{1}}, r_{i 2} \frac{\partial e_{i}(t, x)}{\partial x_{2}}, \ldots, r_{i m} \frac{\partial e_{i}(t, x)}{\partial x_{m}}\right)^{T}
$$


In view of Lemma 2.3, it is derived that

$$
-\sum_{i=1}^{n} \int_{\Omega} \sum_{l=1}^{m} r_{i l}\left(\frac{\partial e_{i}(t, x)}{\partial x_{l}}\right)^{2} \mathrm{~d} x \leq-\sum_{i=1}^{n} \int_{\Omega} \sum_{l=1}^{m} \frac{r_{i l}}{z_{l}^{2}} e_{i}^{2}(t, x) \mathrm{d} x=-\int_{\Omega} e^{T}(t, x) \tilde{R} e(t, x) \mathrm{d} x .
$$

For any positive constants $\varepsilon_{1}, \varepsilon_{2}$, and $\varepsilon_{3}$, it follows from $\left(\mathrm{H}_{1}\right)$ and Lemma 2.4 that

$$
\begin{aligned}
& e^{T}(t, x) A g(e(t, x)) \leq \frac{1}{2} \varepsilon_{1}^{-1} e^{T}(t, x) A A^{T} e(t, x)+\frac{1}{2} \varepsilon_{1} g^{T}(e(t, x)) g(e(t, x)) \\
& \leq \frac{1}{2}\left(\varepsilon_{1}^{-1}\|A\|^{2}+\varepsilon_{1} \mu\right) e^{T}(t, x) e(t, x) \\
& e^{T}(t, x) B g\left(e\left(t-\tau_{1}(t), x\right)\right) \leq \frac{1}{2} \varepsilon_{2}^{-1}\|B\|^{2} e^{T}(t, x) e(t, x)+\frac{1}{2} \varepsilon_{2} \mu e^{T}\left(t-\tau_{1}(t), x\right) e\left(t-\tau_{1}(t), x\right), \\
& e^{T}(t, x) D \int_{-\infty}^{t} K(t-s) g(e(s, x)) \mathrm{d} s \leq \frac{1}{2} \varepsilon_{3}\left(\int_{-\infty}^{t} K(t-s) g(e(s, x)) \mathrm{d} s\right)^{T} \\
& \times \int_{-\infty}^{t} K(t-s) g(e(s, x)) \mathrm{d} s+\frac{1}{2} \varepsilon_{3}^{-1}\|D\|^{2} e^{T}(t, \mathrm{x}) e(t, x) .
\end{aligned}
$$

By using condition $\left(\mathrm{H}_{2}\right)$ and Lemma 2.5, one obtains from (3.12) that

$$
\begin{aligned}
e^{T}(t, x) D \int_{-\infty}^{t} K(t-s) g(e(s, x)) \mathrm{d} s \leq & \frac{1}{2} \varepsilon_{3} \bar{k} \int_{-\infty}^{t} K(t-s) g^{T}(e(s, x)) g(e(s, x)) \mathrm{d} s \\
& +\frac{1}{2} \varepsilon_{3}^{-1}\|D\|^{2} e^{T}(t, x) e(t, x) \\
\leq & \frac{1}{2} \varepsilon_{3} \bar{k} \mu \int_{-\infty}^{t} K(t-s) e^{T}(s, x) e(s, x) \mathrm{d} s \\
& +\frac{1}{2} \varepsilon_{3}^{-1}\|D\|^{2} e^{T}(t, x) e(t, x) .
\end{aligned}
$$
that

Considering condition $\left(\mathrm{H}_{3}\right)$ and substituting (3.9)-(3.11) and (3.13) into (3.6) derive

$$
\begin{aligned}
\varrho V(t) \leq \int_{\Omega}[ & \frac{a}{2} e^{T}(t, x) e(t, x)+\frac{1}{2} \varepsilon_{2} \mu e^{T}\left(t-\tau_{1}(t), x\right) e\left(t-\tau_{1}(t), x\right) \\
& +\frac{\rho_{2}}{2} e^{T}\left(t-\tau_{2}(t), x\right) e\left(t-\tau_{2}(t), x\right)+\frac{1}{2} \varepsilon_{3} \bar{k} \mu \int_{-\infty}^{t} K(t-s) e^{T}(s, x) e(s, x) \mathrm{d} s \\
& \left.+\frac{\rho_{3}}{2} \int_{t-\tau_{3}(t)}^{t} e^{T}(s, x) e(s, x) d s\right] \mathrm{d} x
\end{aligned}
$$




$$
\begin{aligned}
= & a V(t)+\varepsilon_{2} \mu V\left(t-\tau_{1}(t)\right)+\rho_{2} V\left(t-\tau_{2}(t)\right)+\varepsilon_{3} \bar{k} \mu \int_{-\infty}^{t} K(t-s) V(s) \mathrm{d} s \\
& +\rho_{3} \int_{t-\tau_{3}(t)}^{t} V(\mathrm{~s}) \mathrm{d} s .
\end{aligned}
$$

Taking mathematical expectations on both sides of (3.5), it can be derived from inequations (3.14) and $\left(\mathrm{H}_{2}\right)$ that

$$
\begin{aligned}
\frac{\mathrm{d} \mathbb{E}\{V(t)\}}{\mathrm{d} t} \leq & a \mathbb{E}\{V(t)\}+\varepsilon_{2} \mu[\mathbb{E}\{V(t)\}]_{\bar{\tau}_{1}}+\rho_{2}[\mathbb{E}\{V(s)\}]_{\bar{\tau}_{2}}+\varepsilon_{3} \bar{k}^{2} \mu[\mathbb{E}\{V(s)\}]_{-\infty} \\
& +\rho_{3} \bar{\tau}_{3}[\mathbb{E}\{V(s)\}]_{\bar{\tau}_{3}}, \quad t \in\left(t_{k-1}, t_{k}\right], k \in \mathbb{N}_{+}
\end{aligned}
$$

where $[\mathbb{E}\{V(s)\}]_{-\infty}=\max _{s \leq t} \mathbb{E}\{V(s)\}$.

On the other hand, it is obtained from $\left(\mathrm{H}_{4}\right)$ and the second equation of (2.11) that

$$
\begin{aligned}
V\left(t_{k}^{+}\right) & =\int_{\Omega} \frac{1}{2} e^{T}\left(t_{k}^{+}, x\right) e\left(t_{k}^{+}, x\right) \mathrm{d} x=\int_{\Omega} \frac{1}{2} h_{k}^{T}\left(t_{k}, x\right) h_{k}\left(t_{k}, x\right) \mathrm{d} x \\
& \leq \alpha_{k} V\left(t_{k}\right)+\beta_{k}^{1} V\left(t_{k}-\eta_{1}\left(t_{k}\right)\right)+\cdots+\beta_{k}^{q} V\left(t_{k}-\eta_{q}\left(t_{k}\right)\right)+\beta_{k}^{q+1} \int_{t-\eta_{q+1}(t)}^{t} V(s) \mathrm{d} s,
\end{aligned}
$$

which means that

$$
\mathbb{E}\left\{V\left(t_{k}^{+}\right)\right\} \leq \alpha_{k} \mathbb{E}\left\{V\left(t_{k}\right)\right\}+\beta_{k}^{1}\left[\mathbb{E}\left\{V\left(t_{k}\right)\right\}\right]_{\bar{\eta}_{1}}+\cdots+\beta_{k}^{q}\left[\mathbb{E}\left\{V\left(t_{k}\right)\right\}\right]_{\bar{\eta}_{q}}+\beta_{k}^{q+1} \bar{\eta}_{q+1}[V(s)]_{\bar{\eta}_{q+1}} .
$$

By virtue of Lemma 2.7, if the inequalities (3.1) and (3.2) hold, then it follows from (3.15) and (3.17) that there exist constants $M>1$ and $\theta>0$ such that

$$
\mathbb{E}\{V(t)\} \leq \max _{s \leq 0} \mathbb{E}\left\{\left\|\bar{\varphi}_{1}(s, x)\right\|^{2}\right\} M e^{-\theta t}, \quad t \geq 0
$$

By Definition 2.2, the controlled system (2.8) is globally exponentially synchronized with system (2.2) in mean square. This completes the proof.

Note that there are three uncertain positive constants $\varepsilon_{1}, \varepsilon_{2}$, and $\varepsilon_{3}$. Not making a good choice of the three constants may lead to the conservativeness of Theorem 3.1 in practical application. In order to hit off this fault, our next aim is to determine the constants $\varepsilon_{1}, \varepsilon_{2}$, and $\varepsilon_{3}$ such that the conservativeness of Theorem 3.1 can be reduced as much as possible. We present the following Theorem 3.2. 
Theorem 3.2. Suppose that conditions $\left(H_{1}\right)-\left(H_{4}\right)$. Then, under the impulsive controller (2.7), the controlled system (2.8) is globally exponentially synchronized with system (2.2) in mean square if the following inequalities hold

$$
\begin{gathered}
0<b_{k}<1, \quad k \in \mathbb{N}_{+}, \\
\xi_{k}=-\lambda_{\min }(\widetilde{R}+C)+\|A\| \sqrt{\mu}+\|B\| \sqrt{\frac{\mu}{b_{k}}}+\bar{k}\|D\| \sqrt{\frac{\mu}{b_{k}}} \\
+\frac{1}{2}\left(\rho_{1}+\frac{\rho_{2}}{b_{k}}+\frac{\rho_{3} \bar{\tau}_{3}}{b_{k}}+\frac{\ln b_{k}}{t_{k+1}-t_{k}}\right)<0, \quad k \in \mathbb{N}_{+}
\end{gathered}
$$

where $b_{k}=\alpha_{k}+\sum_{i=1}^{q} \beta_{k}^{i}+\beta_{k}^{q+1} \bar{\eta}_{q+1}$, the other parameters are defined as those in Theorem 3.1.

Proof. Define the function $H\left(\varepsilon_{1}, \varepsilon_{2}, \varepsilon_{3}\right)$ with positive variables $\varepsilon_{1}, \varepsilon_{2}$, and $\varepsilon_{3}$ as follows:

$$
H\left(\varepsilon_{1}, \varepsilon_{2}, \varepsilon_{3}\right)=a+\frac{\varepsilon_{2} \mu+\rho_{2}+\varepsilon_{3} \bar{k}^{2} \mu+\rho_{3} \bar{\tau}_{3}}{\alpha_{k}+\sum_{i=1}^{q} \beta_{k}^{i}+\beta_{k}^{q+1} \bar{\eta}_{q+1}}+\frac{\ln \left(\alpha_{k}+\sum_{i=1}^{q} \beta_{k}^{i}\right)+\beta_{k}^{q+1} \bar{\eta}_{q+1}}{t_{k+1}-t_{k}} .
$$

In order that the result of Theorem 3.1 is less conservative, we only need to find out three constants $\varepsilon_{1}^{0}, \varepsilon_{2}^{0}$, and $\varepsilon_{3}^{0}$ such that the inequality (3.2) is less conservative. To achieve this goal, we will find a point $\left(\varepsilon_{1}^{0}, \varepsilon_{2}^{0}, \varepsilon_{3}^{0}\right)$ such that $H\left(\varepsilon_{1}^{0}, \varepsilon_{2}^{0}, \varepsilon_{3}^{0}\right)$ takes the minimum value and $H\left(\varepsilon_{1}^{0}, \varepsilon_{2}^{0}, \varepsilon_{3}^{0}\right)<0$. By simple computation, one derives that $\partial H / \partial \varepsilon_{1}=\mu-\left(\|A\|^{2} / \varepsilon_{1}^{2}\right)$, $\partial H / \partial \varepsilon_{2}=\left(\mu / b_{k}\right)-\left(\|B\|^{2} / \varepsilon_{2}^{2}\right), \partial H / \partial \varepsilon_{3}=\left(\bar{k}^{2} \mu / b_{k}\right)-\left(\|D\|^{2} / \varepsilon_{3}^{2}\right)$. Let $\partial H / \partial \varepsilon_{1}=\partial H / \partial \varepsilon_{2}=$ $\partial H / \partial \varepsilon_{3}=0$, one gets $\left(\varepsilon_{1}^{0}, \varepsilon_{2}^{0}, \varepsilon_{3}^{0}\right)=\left(\|A\| / \sqrt{\mu},\|B\| \sqrt{b_{k} / \mu},\|D\| / \bar{k} \sqrt{b_{k} / \mu}\right)$. It is obvious that the Hesse matrix of $H\left(\varepsilon_{1}, \varepsilon_{2}, \varepsilon_{3}\right)$ at $\left(\varepsilon_{1}^{0}, \varepsilon_{2}^{0}, \varepsilon_{3}^{0}\right)$ is positive definite. Hence, $H\left(\varepsilon_{1}, \varepsilon_{2}, \varepsilon_{3}\right)$ takes the minimum value at $\left(\varepsilon_{1}^{0}, \varepsilon_{2}^{0}, \varepsilon_{3}^{0}\right)$ according to the extreme value theory of multivariate function. Taking $H\left(\varepsilon_{1}^{0}, \varepsilon_{2}^{0}, \varepsilon_{3}^{0}\right)<0$ arrives at the condition (3.20). This completes the proof.

Remark 3.3. Theorems 3.1 and 3.2 are not dependent on discrete delays of both continuous equation and impulsive controller, which is consistent with results of $[3,9,26,38]$, though they did not consider delays in impulses. It should be noted that the inequalities in Theorems 3.1 and 3.2 are related to $\bar{k}^{2}, \bar{\tau}_{3}$, and $\bar{\eta}_{q+1}$, which mean that distributed delays in both continuous equation and impulsive controller have important effects on synchronization criteria in our results. This new discovery is completely different from existing results including those in $[3,9,26,37-40]$. As was pointed out in Remark 2.8, results in $[3,9,38,40]$ were derived by using similar method used in [26], hence results of this paper improve those in $[3,9,26,38,40]$ even when $D=0, \sigma(t, x)=\sigma\left(t, e(t, x), e\left(t-\tau_{2}(t), x\right)\right)$ and $h_{k}\left(t_{k}, x\right)=h\left(e\left(t_{k}, x\right)\right)$ in (2.11). To sum up, results of this paper are new and improve and extend most of known corresponding ones.

Remark 3.4. Lemma 2.5 is utilized in (3.13), which makes the proof process more simple than those in [21, 22, 34]. In [21], matrix decomposition method was used to deal with not-equalto-1 delay kernel, hence the Lyapunov functional and proof process are relatively complex. Authors in $[22,34]$ had to utilize algebraic approach instead of matrix method to derive their main results. It is well known that results derived from algebraic approach have more 
complex form and is more conservative than those obtained by matrix method. Therefore, results of this paper improve those in $[21,22,34]$ to some extent.

Remark 3.5. Stochastic perturbations are unavoidable in real applications of neural networks. In this paper, we synchronize a class of reaction-diffusion neural networks with stochastic perturbations via impulsive control. Although there were several results on stability of reaction-diffusion neural with stochastic perturbations $[45,46]$, seldom published papers considered synchronization of this kind of neural networks under impulsive control. Moreover, the stochastic perturbations of this paper are more general than those in $[45,46]$, since they include information of distributed delays.

\section{Examples and Simulations}

As applications of the the theoretical results derived above, in this section, we give numerical simulations to demonstrate that our synchronization criteria are effective.

Consider the following reaction-diffusion neural network with both discrete and unbounded distributed delays

$$
\begin{aligned}
\frac{\partial y(t, x)}{\partial t}= & \frac{\partial}{\partial x}\left(R \frac{\partial y(t, x)}{\partial x}\right)-C y(t, x)+A f(y(t, x))+B f\left(y\left(t-\tau_{1}(t), x\right)\right) \\
& +D \int_{-\infty}^{t} K(t-s) f(y(s, x)) \mathrm{d} s+I(t)
\end{aligned}
$$

where $y(t, x)=\left(y_{1}(t, x), y_{2}(t, x)\right)^{T}, x \in \Omega=[-2,2]$,

$f(y(t, x))=\left(\tanh \left(x_{1}(t, x)\right), \tanh \left(x_{2}(t, x)\right)\right)^{T}, \tau_{1}(t)=1, K(t)=e^{-0.5 t}, R=\operatorname{diag}(0.1,0.1), I(t)=$ $(1,1.2)^{T}$,

$$
C=\left(\begin{array}{cc}
1.2 & 0 \\
0 & 1
\end{array}\right), \quad A=\left(\begin{array}{cc}
3 & -0.3 \\
4 & 5
\end{array}\right), \quad B=\left(\begin{array}{cc}
-1.4 & 0.1 \\
0.3 & -8
\end{array}\right), \quad D=\left(\begin{array}{cc}
-1.2 & 0.1 \\
-2.8 & -1
\end{array}\right)
$$

Take the boundary condition of $(4.1)$ as $y(t, x)=0,(t, x) \in(-\infty,+\infty) \times \partial \Omega$. In the case that initial condition is chosen as $y(s, x)=(0.4,0.6)^{T},(s, x) \in[-3,0] \times \Omega$ and $y(s, x)=0$, $(s, x) \in(-\infty,-3) \times \Omega$, the chaotic-like trajectory of (4.1) is shown in Figures 1, 2, and 3. Taking $R=0$, then we get the chaotic-like trajectory of (4.1) without reaction-diffusion terms shown in Figure 4.

Let system (4.1) be the driver network, we design a response system as

$$
\begin{gathered}
\mathrm{d} u(t, x)=\left[\frac{\partial}{\partial x}\left(R \frac{\partial u(t, x)}{\partial x}\right)-C u(t, x)+A f(u(t, x))+B f\left(u\left(t-\tau_{1}(t), x\right)\right)\right. \\
\left.+D \int_{-\infty}^{t} K(t-s) f(u(s, x)) \mathrm{d} s+I(t)\right] \mathrm{d} t+\sigma(t, x) \mathrm{d} \omega(t), \quad t \neq t_{k}, \\
u\left(t_{k}^{+}, x\right)=u\left(t_{k}^{-}, x\right)+h_{k}\left(t_{k}, x\right)-e\left(t_{k}, x\right), \quad t=t_{k}, \quad k \in \mathbb{N}_{+},
\end{gathered}
$$




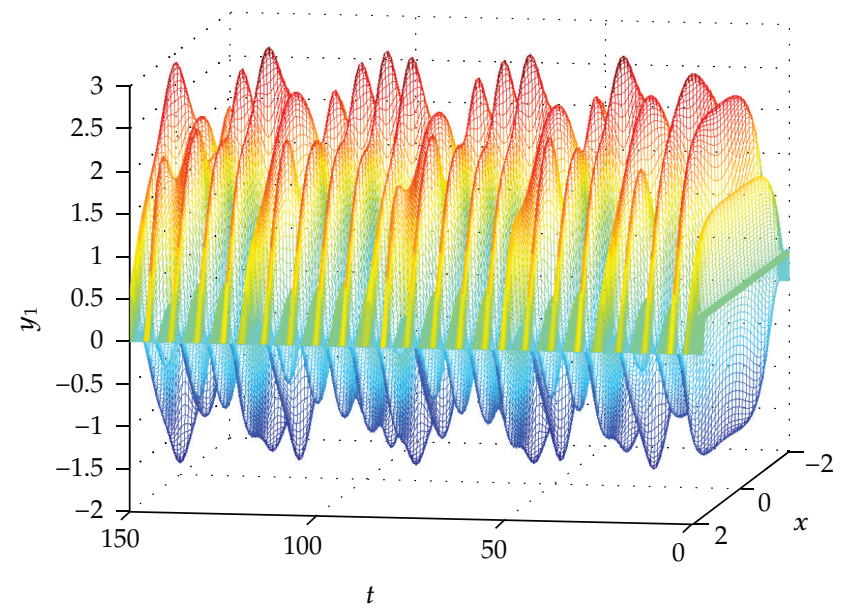

Figure 1: Chaotic behavior of the state $y_{1}(t, x)$ in system (4.1).

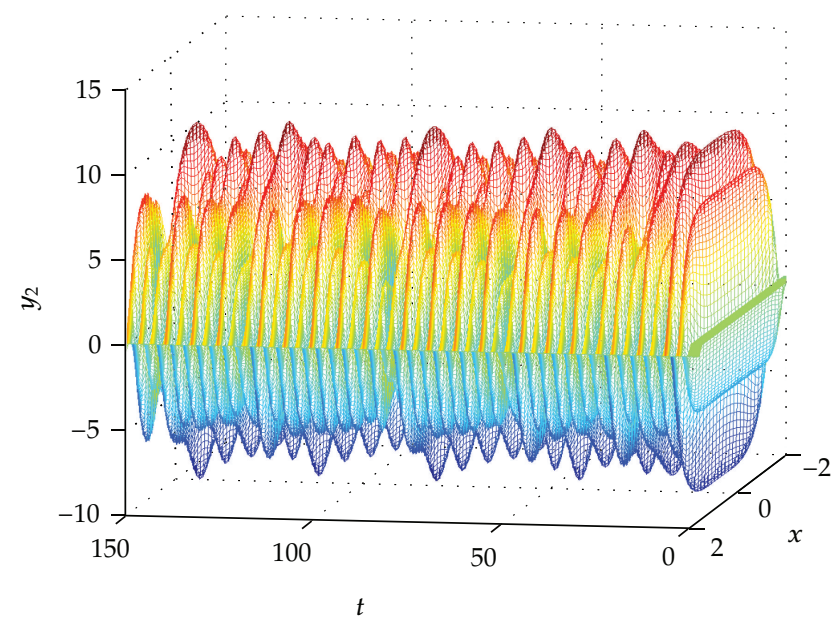

Figure 2: Chaotic behavior of the state $y_{2}(t, x)$ in system (4.1).

where $e(t, x)=u(t, x)-y(t, x), h_{k}\left(t_{k}, x\right)=a e\left(t_{k}, x\right)+b e\left(t_{k}-0.5\left|\sin t_{k}\right|, x\right)+c \int_{t-0.5}^{t} e(s, x) \mathrm{d} s$ with positive constants $a, b$, and $c$, the noise intensity function matrix is

$$
\sigma(t, x)=0.1\left(\begin{array}{cc}
e_{1}(t, x) & e_{2}(t-1, x) \\
\int_{t-0.3}^{t} e_{1}(s, x) \mathrm{d} s & e_{2}(t, x)
\end{array}\right) .
$$

By Jensen's inequality (which is a special case of inequality (2.19)), one has

$$
\left(\int_{t-0.3}^{t} e_{1}(s, x) \mathrm{d} s\right)^{2} \leq 0.3 \int_{t-0.3}^{t}\left(e_{1}(s, x)\right)^{2} \mathrm{~d} s \leq 0.3 \int_{t-0.3}^{t} e^{T}(s, x) e(s, x) \mathrm{d} s .
$$




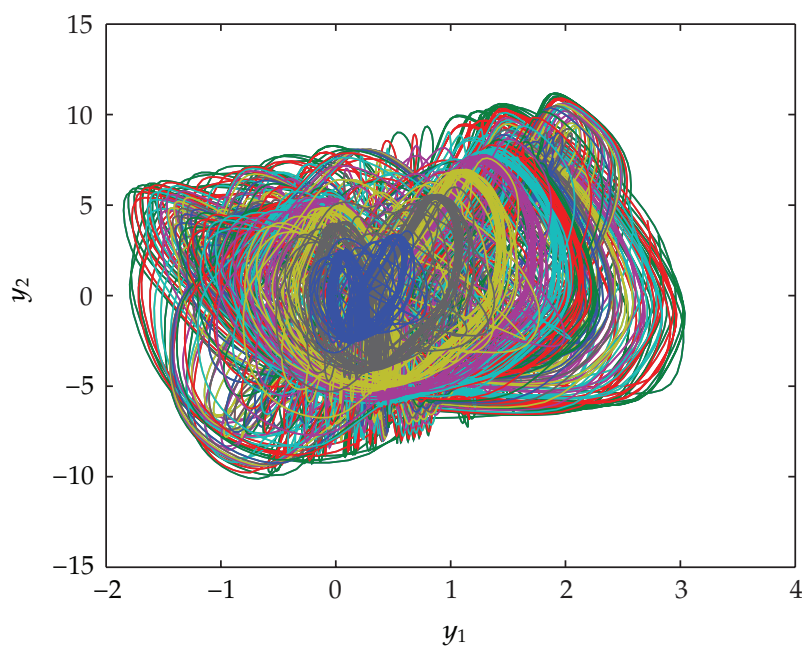

Figure 3: Chaotic behavior of system (4.1).

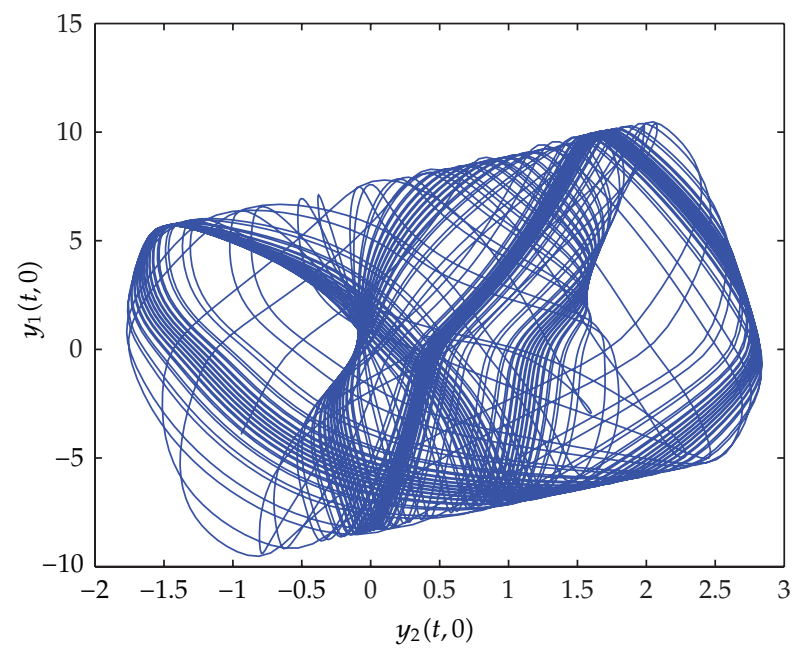

Figure 4: Chaotic behavior of system (4.1) with $R=0$.

From (4.5) one gets

$$
\begin{aligned}
\operatorname{trace}\left(\sigma^{T}(t, x) \sigma(t, x)\right) \leq & 0.01 e^{T}(t, x) e(t, x)+0.01 e^{T}(t-1, x) e(t-1, x) \\
& +0.003 \int_{t-0.3}^{t} e^{T}(s, x) e(s, x) \mathrm{d} s
\end{aligned}
$$




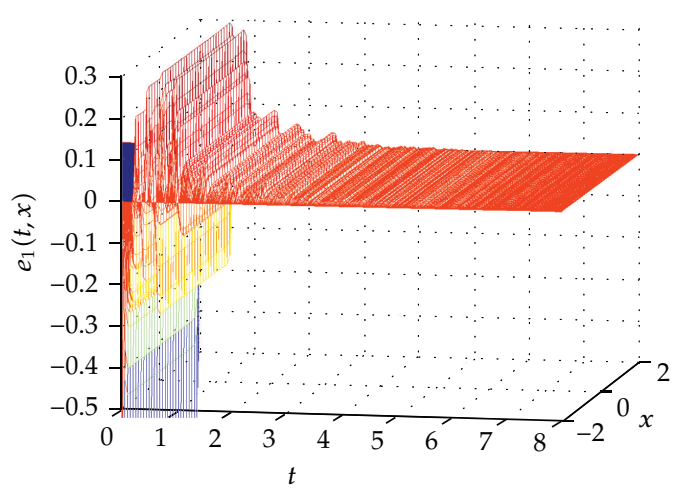

(a)

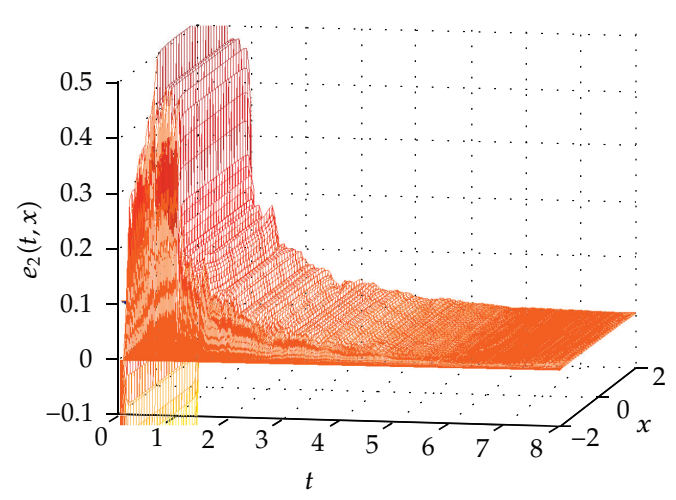

(b)

Figure 5: Dynamical behavior of synchronization errors $e_{1}(t, x)(\mathrm{a})$ and $e_{2}(t, x)(\mathrm{b})$.

Similarly, by using Jensen's inequality one derives that

$$
\begin{gathered}
h_{k}^{T}\left(t_{k}, x\right) h_{k}\left(t_{k}, x\right) \leq(a+b+c)\left[a e^{T}\left(t_{k}, x\right) e\left(t_{k}, x\right)+b e^{T}\left(t_{k}-0.5\left|\sin t_{k}\right|, x\right) e\left(t_{k}-0.5\left|\sin t_{k}\right|, x\right)\right. \\
\left.+c \int_{t-0.5}^{t} e^{T}(s, x) e(s, x) \mathrm{d} s\right] .
\end{gathered}
$$

Obviously, $\mu_{1}=\mu_{2}=1, \bar{k}=2, \rho_{1}=\rho_{2}=0.01, \rho_{3}=0.003, \alpha_{k}=a(a+b+c), \beta_{k}^{1}=b(a+b+c)$, $\beta_{k}^{2}=c(a+b+c), q=1, \bar{\tau}_{1}=\bar{\tau}_{2}=1, \bar{\tau}_{3}=0.3$, and $\bar{\eta}_{1}=\bar{\eta}_{2}=0.5$. Therefore, $\left(\mathrm{H}_{1}\right)-\left(\mathrm{H}_{4}\right)$ are satisfied. Choose $a=0.2, b=0.15, c=0.2$, and $t_{k}-t_{k-1}=0.02$. Then the inequalities (3.19) and (3.20) are satisfied with $b_{k}=0.2475, \xi_{k}=-0.4086<0$, respectively. According to Theorem 3.2, the controlled system (4.3) is globally exponentially synchronized with system (4.1) in mean square. Figure 5 presents the dynamical behavior of synchronization errors $e_{1}(t, x)$ and $e_{2}(t, x)$, which close to zero quickly as time increases.

\section{Conclusion}

Delays are unavoidable in practical systems, and they are always unknown and time-varying. This paper studies stochastic synchronization of reaction-diffusion neural networks with both time-varying discrete and distributed delays via delayed impulsive control. The impulsive controller has multiple time-varying discrete and distributed delays which is very general. Based on a novel integral inequality, the problem of distributed delays with not-equal-to1 delay kernel is well handled with matrix method. Sufficient synchronization criteria are given to guarantee the global exponential synchronization in mean square of the considered system. The function extreme value theorem is utilized to get a less conservative result. It is discovered that, in our synchronization criteria, the distributed delays in both continuous equation and impulsive controller have important effects. At last, numerical simulations show the validity of the obtained criteria. 


\section{Appendix}

Proof of Lemma 2.7. Without loss of generality, we assume that $\tau=\tau_{1} \geq \tau_{2} \geq \cdots \geq \tau_{m}$. Consider the following scalar function:

$$
g_{k}(\lambda)=2 \lambda+a+\frac{\sum_{i=1}^{m} b_{i} e^{\lambda \tau_{i}}}{p_{k}+\sum_{j=1}^{m} q_{k}^{j} e^{\lambda \tau_{j}}}+\frac{\ln \left(p_{k}+\sum_{j=1}^{m} q_{k}^{j} e^{\lambda \tau_{j}}\right)}{t_{k+1}-t_{k}} .
$$

It follows from inequality (2.22) that $g_{k}(0)=a+\left(\sum_{i=1}^{m} b_{i}\right) /\left(p_{k}+\sum_{j=1}^{m} q_{k}^{j}\right)+\left(\ln \left(p_{k}+\right.\right.$ $\left.\left.\sum_{j=1}^{m} q_{k}^{j}\right)\right) /\left(t_{k+1}-t_{k}\right)<0$. Since $g_{k}^{\prime}(\lambda)=2+\sum_{i=1}^{m}\left(p_{k} b_{i} \lambda e^{\lambda \tau_{i}} /\left(p_{k}+\sum_{j=1}^{m} q_{k}^{j} e^{\lambda \tau_{j}}\right)^{2}\right)+$ $\left(\lambda \sum_{j=1}^{m} q_{k}^{j} e^{\lambda \tau_{j}}\right) /\left(\left(t_{k+1}-t_{k}\right)\left(p_{k}+\sum_{j=1}^{m} q_{k}^{j} e^{\lambda \tau_{j}}\right)\right)>0$ for $\lambda>0$ and $g_{k}(\lambda)$ is continuous on $(0,+\infty)$, there exists a positive constant $\lambda$ such that $g_{k}(\lambda)<0$ and $p_{k}+\sum_{j=1}^{m} q_{k}^{j} e^{\lambda \tau_{j}} \leq 1$ for all $k \in \mathbb{N}_{+}$.

Let $\gamma=\sup _{k \in \mathbb{N}_{+}}\left\{1 /\left(p_{k}+\sum_{j=1}^{m} q_{k}^{j} e^{\lambda \tau_{j}}\right)\right\} \geq 1$. Then we can select a constant $\sigma>0$ such that for all $k \in \mathbb{N}_{+}$,

$$
\begin{gathered}
a+\sum_{i=1}^{m} \gamma b_{i} e^{\lambda \tau_{i}} \leq \sigma-\lambda \\
(\sigma+\lambda)\left(t_{k+1}-t_{k}\right)<-\ln \left(p_{k}+\sum_{j=1}^{m} q_{k}^{j} e^{\lambda \tau_{j}}\right) \leq \ln \gamma .
\end{gathered}
$$

From (A.3), we can choose $\beta=\beta_{1} \geq \beta_{2} \geq \cdots \geq \beta_{m}>1$ such that

$$
1<e^{(\sigma+\lambda)\left(t_{1}-t_{0}\right)} \leq \beta_{i} \leq \gamma e^{\lambda \tau_{i}} .
$$

It follows from the above inequality that

$$
\|\phi\|_{\tau}<\|\phi\|_{\tau} e^{\sigma\left(t_{1}-t_{0}\right)} \leq\|\phi\|_{\tau} \beta_{1} e^{-\lambda\left(t_{1}-t_{0}\right)} .
$$

Next we will prove that

$$
v(t) \leq\|\phi\|_{\tau} \beta_{1} e^{-\lambda\left(t-t_{0}\right)}, \quad t \in\left[t_{k-1}, t_{k}\right), \quad k \in \mathbb{N}_{+} .
$$

We use mathematical induction to prove that (A.6) holds. Firstly, we prove that (A.6) holds for $k=1$. To do this, we only need to prove that

$$
v(t) \leq\|\phi\|_{\tau} \beta_{1} e^{-\lambda\left(t_{1}-t_{0}\right)}, \quad t \in\left[t_{0}, t_{1}\right) .
$$

If the inequality (A.7) is not true, then there exists some $\bar{t} \in\left(t_{0}, t_{1}\right)$ such that

$$
\begin{aligned}
v(\bar{t}) & >\|\phi\|_{\tau} \beta_{1} e^{-\lambda\left(t_{1}-t_{0}\right)} \geq\|\phi\|_{\tau} \beta_{2} e^{-\lambda\left(t_{1}-t_{0}\right)} \geq \cdots \geq\|\phi\|_{\tau} \beta_{m} e^{-\lambda\left(t_{1}-t_{0}\right)} \\
& \geq\|\phi\|_{\tau} e^{\sigma\left(t_{1}-t_{0}\right)}>\|\phi\|_{\tau} \geq v\left(t_{0}+s\right), \quad s \in[-\tau, 0]
\end{aligned}
$$


which implies that there exists $\tilde{t}_{i} \in\left(t_{0}, \bar{t}\right)$ such that $\tilde{t}_{m} \leq \tilde{t}_{m-1} \leq \cdots \leq \tilde{t}_{1}$ and

$$
v\left(\tilde{t}_{i}\right)=\|\phi\|_{\tau} \beta_{i} e^{-\lambda\left(t_{1}-t_{0}\right)}, \quad v(t) \leq v\left(\tilde{t}_{i}\right), \quad t \in\left[t_{0}-\tau, \tilde{t}_{i}\right]
$$

and there exists $\hat{t} \in\left[t_{0}, \tilde{t}_{m}\right)$ such that

$$
v(\hat{t})=\|\phi\|_{\tau^{\prime}} \quad v(\hat{t}) \leq v(t) \leq v\left(\tilde{t}_{i}\right), \quad t \in\left[\widehat{t}, \tilde{t}_{i}\right]
$$

Therefore, one gets from (A.4), (A.9), and (A.10) that, for any $s \in\left[-\tau_{i}, 0\right]$,

$$
v(t+s) \leq\|\phi\|_{\tau} \beta_{i} e^{-\lambda\left(t_{1}-t_{0}\right)} \leq\|\phi\|_{\tau} \gamma e^{\lambda \tau_{i}} e^{-\lambda\left(t_{1}-t_{0}\right)} \leq \gamma e^{\lambda \tau_{i}} v(\widehat{t}) \leq \gamma e^{\lambda \tau_{i}} v(t), \quad t \in\left[\widehat{t}, \tilde{t}_{i}\right] .
$$

Thus, one has from (A.2) and (A.11) that

$$
\begin{aligned}
D^{+} v(t) & \leq a v(t)+b_{1}[v(t)]_{\tau_{1}}+b_{2}[v(t)]_{\tau_{2}}+\cdots+b_{m}[v(t)]_{\tau_{m}} \\
& \leq\left(a+\sum_{i=1}^{m} r b_{i} e^{\lambda \tau_{i}}\right) v(t) \leq(\sigma-\lambda) v(t), \quad t \in\left[\widehat{t}, \tilde{t}_{1}\right] .
\end{aligned}
$$

It follows from (A.5), (A.9), (A.10), and (A.12) that

$$
\begin{aligned}
v\left(\tilde{t}_{1}\right) & \leq v(\hat{t}) e^{(\sigma-\lambda)\left(\widetilde{t}_{1}-\hat{t}\right)}=\|\phi\|_{\tau} e^{(\sigma-\lambda)\left(\tilde{t}_{1}-\hat{t}\right)}<\|\phi\|_{\tau} e^{\sigma\left(t_{1}-t_{0}\right)} \\
& \leq\|\phi\|_{\tau} \beta_{1} e^{-\lambda\left(t_{1}-t_{0}\right)}=v\left(\widetilde{t}_{1}\right)
\end{aligned}
$$

which is a contradiction. Hence (A.6) holds for $k=1$.

Now we assume that (A.6) holds for $k=1,2, \ldots, n, n \in \mathbb{N}_{+}, n \geq 1$, that is,

$$
v(t) \leq\|\phi\|_{\tau} \beta_{1} e^{-\lambda\left(t-t_{0}\right)}, \quad t \in\left[t_{k-1}, t_{k}\right), \quad k=1,2, \ldots, n .
$$

Next, we will show that (A.6) holds for $k=n+1$, that is,

$$
v(t) \leq\|\phi\|_{\tau} \beta_{1} e^{-\lambda\left(t-t_{0}\right)}, \quad t \in\left[t_{n}, t_{n+1}\right) .
$$


For the sake of contradiction, suppose that (A.15) does not hold. Define $\breve{t}=\inf \{t \in$ $\left.\left[t_{n}, t_{n+1}\right] \mid v(t)>\|\phi\|_{\tau} \beta_{1} e^{-\lambda\left(t-t_{0}\right)}\right\}$. Then one obtains from (A.3) and (A.14) that

$$
\begin{aligned}
& v\left(t_{n}^{+}\right) \leq p_{n} v\left(t_{n}^{-}\right)+q_{n}^{1}\left[v\left(t_{n}^{-}\right)\right]_{\tau_{1}}+q_{n}^{2}\left[v\left(t_{n}^{-}\right)\right]_{\tau_{2}}+\cdots+q_{n}^{m}\left[v\left(t_{n}^{-}\right)\right]_{\tau_{m}} \\
& \leq p_{n}\|\phi\|_{\tau} \beta_{1} e^{-\lambda\left(t_{n}-t_{0}\right)}+q_{n}^{1}\|\phi\|_{\tau} \beta_{1} e^{-\lambda\left(t_{n}-\tau_{1}-t_{0}\right)}+q_{n}^{2}\|\phi\|_{\tau} \beta_{1} e^{-\lambda\left(t_{n}-\tau_{2}-t_{0}\right)} \\
& +\cdots+q_{n}^{m}\|\phi\|_{\tau} \beta_{1} e^{-\lambda\left(t_{n}-\tau_{m}-t_{0}\right)} \\
& =\left(p_{n}+\sum_{j=1}^{m} q_{n}^{j} e^{\lambda \tau_{j}}\right)\|\phi\|_{\tau} \beta_{1} e^{\lambda\left(\breve{t}-t_{n}\right)} e^{-\lambda\left(\breve{t}-t_{0}\right)} \\
& <\left(p_{n}+\sum_{j=1}^{m} q_{n}^{j} e^{\lambda \tau_{j}}\right) e^{\lambda\left(t_{n+1}-t_{n}\right)}\|\phi\|_{\tau} \beta_{1} e^{-\lambda\left(\tilde{t}-t_{0}\right)} \\
& <e^{-(\sigma+\lambda)\left(t_{n+1}-t_{n}\right)} e^{\lambda\left(t_{n+1}-t_{n}\right)}\|\phi\|_{\tau} \beta_{1} e^{-\lambda\left(t\left(t-t_{0}\right)\right.} \\
& =e^{-\sigma\left(t_{n+1}-t_{n}\right)}\|\phi\|_{\tau} \beta_{1} e^{-\lambda\left(\tilde{t}-t_{0}\right)}<\|\phi\|_{\tau} \beta_{1} e^{-\lambda\left(t-t_{0}\right)},
\end{aligned}
$$

which implies that $\breve{t} \neq t_{n}$. From the continuity of $v(t)$ in the interval $\left[t_{n}, t_{n+1}\right)$, one has

$$
v(\breve{t})=\|\phi\|_{\tau} \beta_{1} e^{-\lambda\left(\breve{t}-t_{0}\right)}, \quad v(t) \leq v(\breve{t}), t \in\left[t_{n}, \breve{t}\right] .
$$

On the other hand, one can deduce from (A.16) that there exists $t^{*} \in\left(t_{n}, \breve{t}\right)$ such that

$$
v\left(t^{*}\right)=\left(p_{n}+\sum_{j=1}^{m} q_{n}^{j} e^{\lambda \tau_{j}}\right) e^{\lambda\left(t_{n+1}-t_{n}\right)}\|\phi\|_{\tau} \beta_{1} e^{-\lambda\left(\breve{t}-t_{0}\right)}, \quad v\left(t^{*}\right) \leq v(t) \leq v(\breve{t}), t \in\left[t^{*}, \breve{t}\right] .
$$

For any $t \in\left[t^{*}, \breve{t}\right], s \in\left[-\tau_{i}, 0\right]$, either $t+s \in\left[t_{0}-\tau_{i}, t_{n}\right)$ or $t+s \in\left[t_{n}, \breve{t}\right]$. Two cases will be discussed as follows.

Case 1. If $t+s \in\left[t_{0}-\tau_{i}, t_{n}\right)$, then one obtains from (A.14) that

$$
\begin{aligned}
v(t+s) & \leq\|\phi\|_{\tau} \beta_{1} e^{-\lambda\left(t-t_{0}\right)} e^{-\lambda s} \leq\|\phi\|_{\tau} \beta_{1} e^{-\lambda\left(\tilde{t}-t_{0}\right)} e^{\lambda(t \bar{t}-t)} e^{\lambda \tau_{i}} \\
& \leq\|\phi\|_{\tau} \beta_{1} e^{-\lambda\left(t^{\prime}-t_{0}\right)} e^{\lambda\left(t_{n+1}-t_{n}\right)} e^{\lambda \tau_{i}} .
\end{aligned}
$$

Case 2. If $t+s \in\left[t_{n}, \breve{t}\right]$, then it follows from (A.17) that

$$
v(t+s) \leq\|\phi\|_{\tau} \beta_{1} e^{-\lambda\left(\breve{t}-t_{0}\right)} \leq\|\phi\|_{\tau} \beta_{1} e^{-\lambda\left(\breve{t}-t_{0}\right)} e^{\lambda\left(t_{n+1}-t_{n}\right)} e^{\lambda \tau_{i}} .
$$


In any case, one has from (A.18), (A.19), and (A.20) that, for any $s \in\left[-\tau_{i}, 0\right]$,

$$
\begin{aligned}
v(t+s) & \leq\|\phi\|_{\tau} \beta_{1} e^{-\lambda\left(\breve{t}-t_{0}\right)} e^{\lambda\left(t_{n+1}-t_{n}\right)} e^{\lambda \tau_{i}}=\frac{e^{\lambda \tau_{i}}}{p_{n}+\sum_{j=1}^{m} q_{n}^{j} e^{\lambda \tau_{j}}} v\left(t^{*}\right) \\
& \leq \frac{e^{\lambda \tau_{i}}}{p_{n}+\sum_{j=1}^{m} q_{n}^{j} e^{\lambda \tau_{j}}} v(t) \leq \gamma e^{\lambda \tau_{i}} v(t), \quad t \in\left[t^{*}, \check{t}\right] .
\end{aligned}
$$

Hence, one obtains from (A.2) and (A.21) that

$$
\begin{aligned}
D^{+} v(t) & \leq a v(t)+b_{1}[v(t)]_{\tau_{1}}+b_{2}[v(t)]_{\tau_{2}}+\cdots+b_{m}[v(t)]_{\tau_{m}} \\
& \leq\left(a+\sum_{i=1}^{m} \gamma b_{i} e^{\lambda \tau_{i}}\right) v(t) \leq(\sigma-\lambda) v(t), \quad t \in\left[t^{*}, \breve{t}\right] .
\end{aligned}
$$

It follows from inequalities (A.3), (A.17), (A.18), and (A.19) that

$$
\begin{aligned}
v(\breve{t}) & \leq v\left(t^{*}\right) e^{(\sigma-\lambda)\left(\breve{t}-t^{*}\right)} \\
& =\left(p_{n}+\sum_{j=1}^{m} q_{n}^{j} e^{\lambda \tau_{j}}\right) e^{\lambda\left(t_{n+1}-t_{n}\right)}\|\phi\|_{\tau} \beta_{1} e^{-\lambda\left(\breve{t}-t_{0}\right)} e^{(\sigma-\lambda)\left(\breve{t}-t^{*}\right)} \\
& <e^{-(\sigma+\lambda)\left(t_{n+1}-t_{n}\right)} e^{\lambda\left(t_{n+1}-t_{n}\right)}\|\phi\|_{\tau} \beta_{1} e^{-\lambda\left(\breve{t}-t_{0}\right)} e^{(\sigma-\lambda)\left(\breve{t}-t^{*}\right)} \\
& =e^{-\sigma\left(t_{n+1}-t_{n}\right)}\|\phi\|_{\tau} \beta_{1} e^{-\lambda\left(\breve{t}-t_{0}\right)} e^{(\sigma-\lambda)\left(\breve{t}-t^{*}\right)} \\
& \leq\|\phi\|_{\tau} \beta_{1} e^{-\lambda\left(\breve{t}-t_{0}\right)}=v(\breve{t})
\end{aligned}
$$

which is a contradiction. Therefore the assumption that the inequality (A.15) does not hold is not true, and hence the inequality (A.6) holds for $k=n+1$. According to the theory of mathematical induction method, the inequality (A.6) holds for all $k \in \mathbb{N}_{+}$. This completes the proof.

\section{Acknowledgments}

This work was jointly supported by the National Natural Science Foundation of China (NSFC) under Grants no. 61263020, no. 11101053, and no. 10971240, the Scientific Research Fund of Yunnan Province under Grant no. 2010ZC150, the Scientific Research Fund of Chongqing Normal University under Grants no. 940115 and 12XLB031, the Key Project of Chinese Ministry of Education (211118), the Excellent Youth Foundation of Educational Committee of Hunan Provincial (10B002), Key Project of Chinese Education Ministry under Grant no. 212138, Natural Science Foundation of Chongqing under Grant CQ CSTC 2011BB0117, and Foundation of Science and Technology project of Chongqing Education Commission under Grant KJ120630. 


\section{References}

[1] L. M. Pecora and T. L. Carroll, "Synchronization in chaotic systems," Physical Review Letters, vol. 64, no. 8, pp. 821-824, 1990.

[2] Y. Wang, T. Bian, J. Xiao, and Y. Huang, "Robust synchronization of complex switched networks with parametric uncertainties and two types of delays," International Journal of Robust and Nonlinear Control. In press.

[3] X. Yang, J. Cao, and J. Lu, "Synchronization of delayed complex dynamical networks with impulsive and stochastic effects," Nonlinear Analysis: Real World Applications, vol. 12, no. 4, pp. 2252-2266, 2011.

[4] Y.-W. Wang, J.-W. Xiao, and H. O. Wang, "Global synchronization of complex dynamical networks with network failures," International Journal of Robust and Nonlinear Control, vol. 20, no. 15, pp. 16671677, 2010.

[5] P. Zhou and R. Ding, "Modified function projective synchronization between different dimension fractional-order chaotic systems," Abstract and Applied Analysis, vol. 2012, Article ID 862989, 2012.

[6] T. L. Liao and N. S. Huang, "An observer-based approach for chaotic synchronization with applications to secure communications," IEEE Transactions on Circuits and Systems I, vol. 46, no. 9, pp. 1144-1150, 1999.

[7] Y. Wang, J. Xiao, C. Wen, and Z. Guan, "Synchronization of continuous dynamical networks with discrete-time communications," IEEE Transactions on Neural Networks, vol. 22, no. 12, pp. 1979-1986, 2011.

[8] S. H. Strogatz and I. Stewart, "Coupled oscillators and biological synchronization," Scientific American, vol. 269, no. 6, pp. 102-109, 1993.

[9] X. Yang, J. Cao, and J. Lu, "Stochastic synchronization of complex networks with nonidentical nodes via hybrid adaptive and impulsive control," IEEE Transactions on Circuits and Systems. I., vol. 59, no. 2, pp. 371-384, 2012.

[10] Y.-W. Wang, H. O. Wang, J.-W. Xiao, and Z.-H. Guan, “Synchronization of complex dynamical networks under recoverable attacks," Automatica, vol. 46, no. 1, pp. 197-203, 2010.

[11] X. Yang and J. Cao, "Stochastic synchronization of coupled neural networks with intermittent control," Physics Letters A, vol. 373, no. 36, pp. 3259-3272, 2009.

[12] J. Cao, Z. Wang, and Y. Sun, "Synchronization in an array of linearly stochastically coupled networks with time delays," Physica A, vol. 385, no. 2, pp. 718-728, 2007.

[13] J. Liang, Z. Wang, and X. Liu, “On synchronization of coupled delayed neural networks," Recent Advances in Nonlinear Dynamics and Synchronization, pp. 117-149, 2009.

[14] J. Lu, D. W. C. Ho, and Z. Wang, "Pinning stabilization of linearly coupled stochastic neural networks via minimum number of controllers," IEEE Transactions on Neural Networks, vol. 20, no. 10, pp. 1617$1629,2009$.

[15] P. Balasubramaniam, R. Chandran, and S. J. S. Theesar, "Synchronization of chaotic nonlinear continuous neural networks with time-varying delay," Cognitive Neurodynamics, vol. 5, pp. 361-371, 2011.

[16] P. Balasubramaniam and C. Vidhya, "Exponential stability of stochastic reaction-diffusion uncertain fuzzy neural networks with mixed delays and Markovian jumping parameters," Expert Systems With Applications, vol. 39, pp. 3109-3115, 2012.

[17] J. G. Lu, "Global exponential stability and periodicity of reaction-diffusion delayed recurrent neural networks with Dirichlet boundary conditions," Chaos, Solitons and Fractals, vol. 35, no. 1, pp. 116-125, 2008.

[18] K. Wang, Z. Teng, and H. Jiang, "Adaptive synchronization in an array of linearly coupled neural networks with reaction-diffusion terms and time delays," Communications in Nonlinear Science and Numerical Simulation, vol. 17, no. 10, pp. 3866-3875, 2012.

[19] X. Liu, "Synchronization of linearly coupled neural networks with reaction-diffusion terms and unbounded time delays," Neurocomputing, vol. 73, no. 13, pp. 2681-2688, 2010.

[20] Q. Gan, R. Xu, and P. Yang, "Exponential synchronization of stochastic fuzzy cellular neural networks with time delay in the leakage term and reaction-diffusion," Communications in Nonlinear Science and Numerical Simulation, vol. 17, no. 4, pp. 1862-1870, 2012.

[21] Z. Wang and H. Zhang, "Global asymptotic stability of reaction-diffusion cohen-grossberg neural networks with continuously distributed delays," IEEE Transactions on Neural Networks, vol. 21, no. 1, pp. 39-49, 2010.

[22] C. Hu, H. Jiang, and Z. Teng, "Impulsive control and synchronization for delayed neural networks with reaction-diffusion terms," IEEE Transactions on Neural Networks, vol. 21, no. 1, pp. 67-81, 2010. 
[23] Y. Lv, W. Lv, and J. Sun, "Convergence dynamics of stochastic reaction-diffusion recurrent neural networks in continuously distributed delays," Nonlinear Analysis: Real World Applications, vol. 9, no. 4, pp. 1590-1606, 2008.

[24] S. Niu, H. Jiang, and Z. Teng, "Boundedness and exponential stability for nonautonomous fenns with distributed delays and reaction-diffusion terms," Neurocomputing, vol. 73, no. 16-18, pp. 2913-2919, 2010.

[25] L. Wan and Q. Zhou, "Exponential stability of stochastic reaction-diffusion Cohen-Grossberg neural networks with delays," Applied Mathematics and Computation, vol. 206, no. 2, pp. 818-824, 2008.

[26] Z. Yang and D. Xu, "Stability analysis and design of impulsive control systems with time delay," IEEE Transactions on Automatic Control, vol. 52, no. 8, pp. 1448-1454, 2007.

[27] S. Jeeva Sathya Theesar, R. Chandran, and P. Balasubramaniam, "Delay-dependent exponential synchronization criteria for chaotic neural networks with time-varying delays," Brazilian Journal of Physics, vol. 42, pp. 207-218, 2012.

[28] G. Zhang, T. Wang, T. Li, and S. Fei, “Exponential synchronization for delayed chaotic neural networks with nonlinear hybrid coupling," Neurocomputing, vol. 85, pp. 53-61, 2012.

[29] P. Balasubramaniam and V. Vembarasan, "Synchronization of recurrent neural networks with mixed time-delays via output coupling with delayed feedback," Nonlinear Dynamics, vol. 70, pp. 667-691, 2012.

[30] Q. Gan, R. Xu, and X. Kang, "Synchronization of chaotic neural networks with mixed time delays," Communications in Nonlinear Science and Numerical Simulation, vol. 16, no. 2, pp. 966-974, 2011.

[31] Q. Song, "Synchronization analysis of coupled connected neural networks with mixed time delays," Neurocomputing, vol. 72, no. 16-18, pp. 3907-3914, 2009.

[32] K. Q. Gu, V. L. Kharitonov, and J. Chen, Stability of Time-Delay Systems, Birkhauser, Cambridge, Mass, USA, 2003.

[33] X. Yang, C. Huang, D. Zhang, and Y. Long, "Dynamics of Cohen-Grossberg neural networks with mixed delays and impulses," Abstract and Applied Analysis, vol. 2008, Article ID 432341, 14 pages, 2008.

[34] X. Nie and J. Cao, "Multistability of competitive neural networks with time-varying and distributed delays," Nonlinear Analysis: Real World Applications, vol. 10, no. 2, pp. 928-942, 2009.

[35] X. Yang, J. Cao, and J. Lu, "Synchronization of coupled neural networks with random coupling strengths and mixed probabilistic time-varying delays," International Journal of Robust and Nonlinear Control. In press.

[36] Y.-W. Wang, M. Yang, H. O. Wang, and Z.-H. Guan, "Robust stabilization of complex switched networks with parametric uncertainties and delays via impulsive control," IEEE Transactions on Circuits and Systems. I., vol. 56, no. 9, pp. 2100-2108, 2009.

[37] H. Jiang and Q. Bi, "Impulsive synchronization of networked nonlinear dynamical systems," Physics Letters A, vol. 374, no. 27, pp. 2723-2729, 2010.

[38] Z.-H. Guan, Z.-W. Liu, G. Feng, and Y.-W. Wang, “Synchronization of complex dynamical networks with time-varying delays via impulsive distributed control," IEEE Transactions on Circuits and Systems. I., vol. 57, no. 8, pp. 2182-2195, 2010.

[39] J. Lu, D. W. C. Ho, and J. Cao, "A unified synchronization criterion for impulsive dynamical networks," Automatica, vol. 46, no. 7, pp. 1215-1221, 2010.

[40] M. Yang, Y.-W. Wang, J.-W. Xiao, and H. O. Wang, "Robust synchronization of impulsively-coupled complex switched networks with parametric uncertainties and time-varying delays," Nonlinear Analysis: Real World Applications, vol. 11, no. 4, pp. 3008-3020, 2010.

[41] X. Wang and D. Xu, "Global exponential stability of impulsive fuzzy cellular neural networks with mixed delays and reaction-diffusion terms," Chaos, Solitons E Fractals, vol. 42, no. 5, pp. 2713-2721, 2009.

[42] Y. Y. Wang, L. Xie, and C. E. de Souza, "Robust control of a class of uncertain nonlinear systems," Systems $\mathcal{E}$ Control Letters, vol. 19, no. 2, pp. 139-149, 1992.

[43] Q. Wu, J. Zhou, and L. Xiang, "Impulses-induced exponential stability in recurrent delayed neural networks," Neurocomputing, vol. 74, no. 17, pp. 3204-3211, 2011.

[44] D. Yue, E. Tian, Y. Zhang, and C. Peng, "Delay-distribution-dependent robust stability of uncertain systems with time-varying delay," International Journal of Robust and Nonlinear Control, vol. 19, no. 4, pp. 377-393, 2009.

[45] P. Balasubramaniam and C. Vidhya, "Global asymptotic stability of stochastic BAM neural networks with distributed delays and reaction-diffusion terms," Journal of Computational and Applied Mathematics, vol. 234, no. 12, pp. 3458-3466, 2010. 
[46] T. Huang, C. Li, S. Duan, and J. A. Starzyk, "Robust exponential stability of uncertain delayed neural networks with stochastic perturbation and impulse effects," IEEE Transactions on Neural Networks and Learning Systems, vol. 23, no. 6, pp. 866-875, 2012. 


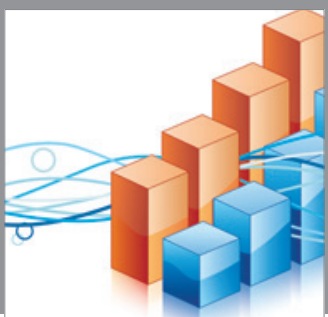

Advances in

Operations Research

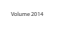

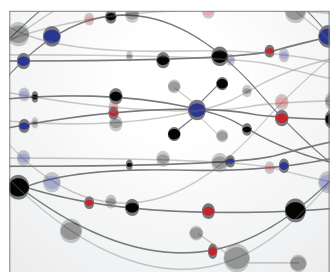

\section{The Scientific} World Journal
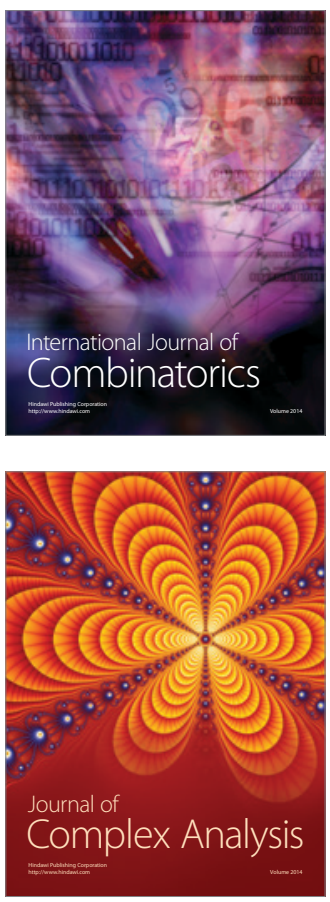

International Journal of

Mathematics and

Mathematical

Sciences
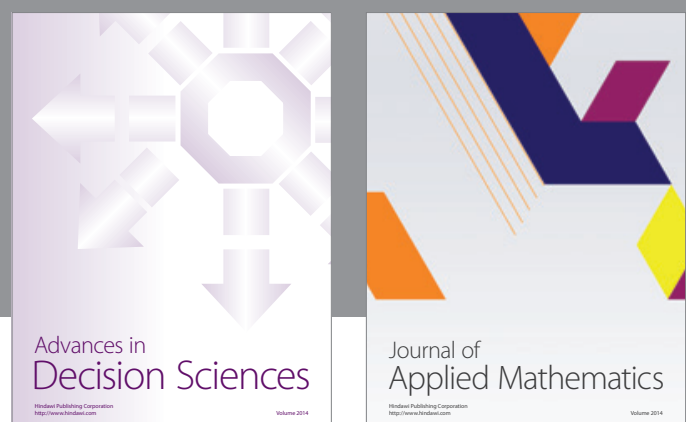

Journal of

Applied Mathematics
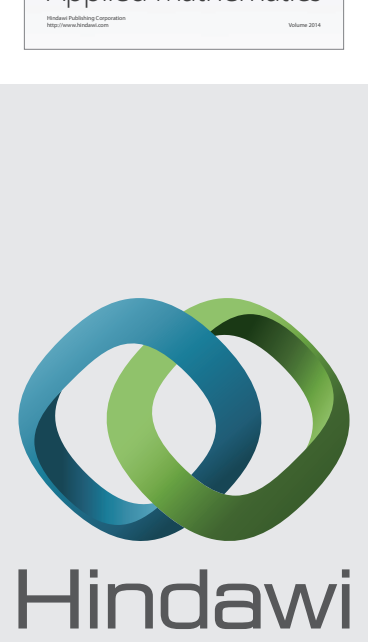

Submit your manuscripts at http://www.hindawi.com
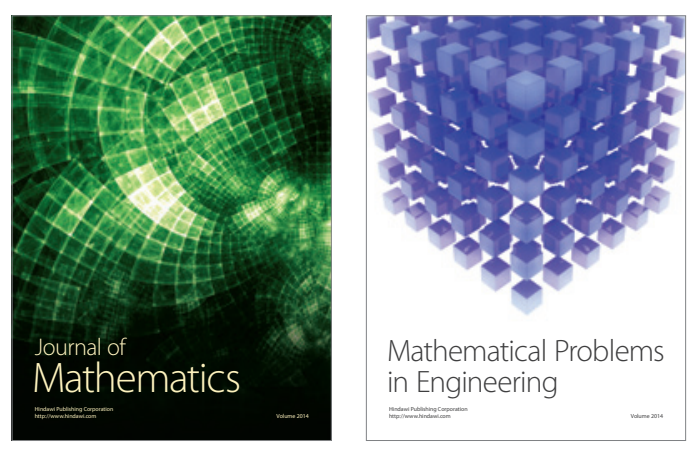

Mathematical Problems in Engineering
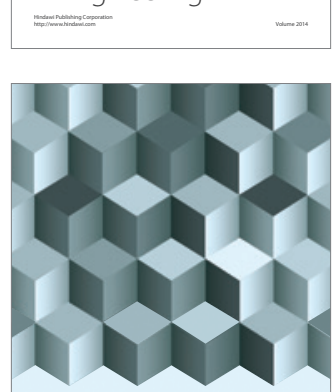

Journal of

Function Spaces
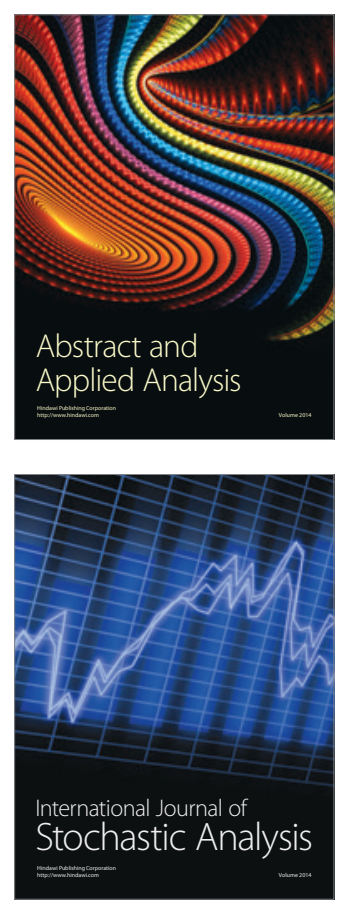

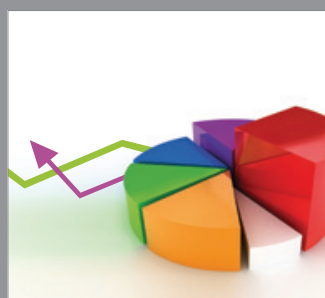

ournal of

Probability and Statistics

Promensencen
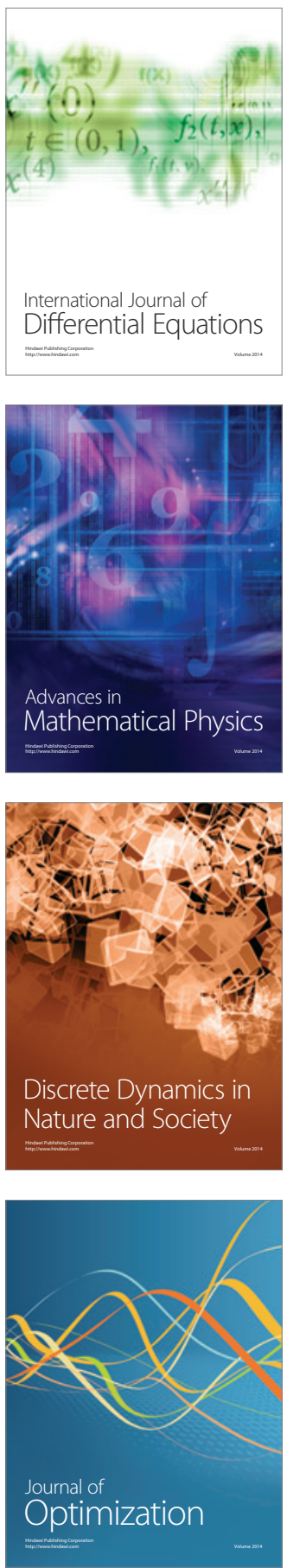